\title{
Interaction of silicone hydrogel contact lenses with lipids - a chronological review
}

\section{Tomasz Suliński ${ }^{1,2}$, Jacek Pniewski}

${ }^{1}$ Alcon Polska, Poland

2 Faculty of Physics, University of Warsaw, Poland Head of Faculty: prof. dr hab. Dariusz Wasik

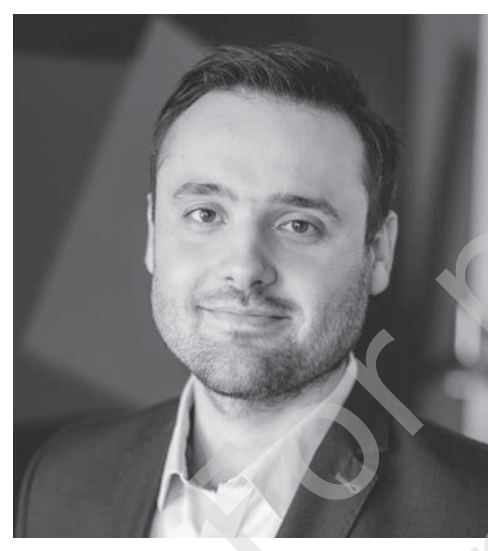

H I G H LI GHTS

Soft contact lenses are a popular method of correcting refractive errors. Silicone hydrogel lenses are becoming more and more popular. Lenses are exposed to interactions with lipids (e.g., from tear film). It is important for eye care practitioners to understand these interactions and how they can affect the patient's clinical response.

\begin{abstract}
Silicone hydrogel (SiHy) contact lenses are a common form of correction of refractive errors prescribed by eye care professionals around the world. SiHy lenses perform in a complex environment, which is the surface of the eye and the tear film. Therefore, they are exposed to various factors, such as lipid deposits. The aims of this paper are to review available scientific reports on the study of SiHy lens interactions with lipids and search for further research objectives. A total of 57 publications were identified and reviewed, from 2003 to 2020. In general, $\mathrm{SiHy}$ lenses are more likely to accumulate lipid deposits than traditional hydrogel lenses, although there are significant differences between $\mathrm{SiHy}$ lens materials that may result from different methods used in the studies. The review includes studies on various aspects of interactions between lenses and lipids, such as those concerning the effectiveness of lipids removal from lenses by care solutions. The conclusion points out future research directions, such as measurements of lipid diffusion in SiHy lens' matrices.
\end{abstract}

Key words: contact lenses, contact lens deposits, silicone hydrogel contact lenses, contact lens discomfort 


\section{INTRODUCTION}

Silicone hydrogel (Si-Hy) contact lenses are a popular method of correcting refractive errors. Together with the older generation of hydrogel contact lenses they create the soft lenses group. They are medical devices, so are formally required to be biocompatible. $\mathrm{Si}$-Hy contact lenses are classified by the U.S. Food and Drug Administration (FDA) as V material group (Silicone Hydrogel Polymers) [1]. A global survey (from 25 countries) showed that $\mathrm{Si}$-Hy lenses were prescribed in $57 \%$ cases out of all contact lens fits (64\% of all soft lenses) in 2019 [2]. The introduction of Si-Hy lenses onto the market in 1998 is considered to be the most significant advancement in contact lens technology since the development of (hydroxyethyl)methacrylate (HEMA) lenses [1].

The biggest advantage of Si-Hy lenses compared to older generation of hydrogel lenses is their high oxygen permeability [1]. However, silicone macromeres integrated into the lens material, responsible for this property, also make the material more hydrophobic.

The environment in which a contact lens is used is the eye surface, where it interacts with a tear film. The tear film is composed of water with substances like mucins, lipids, proteins, and electrolytes. A recent study found that an older structural model (3-layer: mucin, aqueous and lipid) of the tear film does not reflect reality, and that the tear film must be considered as a single dynamic functional unit with different compartments [3]. Particularly, the outer lipid layer was described as a bilayer consisting of an upper non- polar layer and a lower polar layer [4]. This description was then extended to "multilamellar sandwich model" where the outer, thick tear lipid layer is created by long, saturated chains of wax esters (WE), cholesterol esters (CE) and other non-polar components such as triacylglycerols (TAG), diesters, free sterols, free fatty acids. There is also an upper no-polar lipid interface between non-polar lipids and the aqueous layer [5]. Most of the lipids in the tear film come from Meibomian glands. Their role is to stabilize and prevent the tear film from evaporating [6-8]. Classes and proportions of the lipids from tear film and meibum are similar (with the exceptions of phospholipids) [9-11]. Main lipid omponents of tears (mol\%) are: cholesterol-ester, wax ester and cholesterol [10].

A number of studies found that $\mathrm{Si}-\mathrm{Hy}$ lenses accumulate more lipid deposits when compared to hydrogel lenses [12, 13]. The Si-Hy lenses are most often contaminated with ipids such as cholesteryl esters, cholesterol and tri-glyceride. These deposits can cause dewetting of a lens surface and lead to tear film instability [14-16], which in turn triggers contact lenses discomfort and may lead to the lenses dropping out [17].
While many studies have been published over the last 20 years, there is still no clear and unanimous information about this process. Therefore, it is important for eye care practitioners to track the research in this field.

\section{PURPOSE}

The aim of this paper it to review available scientific reports on the study of Si-Hy lens interactions with lipids; present available knowledge, results, conclusions and search for further research objectives.

\section{METHODS}

The publications have been obtained from the PubMed database (www.ncbi.nlm.nih.gov/pubmed). The phrase used in the search was "lipid contact lenses". The search was restricted in time-frame (from 2003 to 2020). The search revealed 336 results. The next step was to look through the publications in order to verify which touched on the subject. In addition, the literature from the reviewed publications was browsed to complement the review with studies that did not appear in the initial search. Altogether 57 publications were identified. Then, the studies were arranged in chronological order and described, presenting the most important conclusions.

\section{RESULTS: A CHRONOLOGICA L REVIEW}

\section{0-2002}

The earliest available studies about lipid deposits on contact lenses are from the 70's [18-21] and 80's [22-29] of the $20^{\text {th }}$ century. These studies were continued and expanded in the 90's [30-41], but they only involved hydrogel lenses. These lenses were tested both in vitro and ex vivo. The research whose review is the aim of this publication began in the 21st century along with the appearance of Si-Hy lenses on the market.

\section{3}

The first scientific paper on lipid deposits on Si-Hy lenses was published. Jones et al. studied deposits of lysozyme and lipids on worn Si-Hy lenses (balafilcon and lotrafilcon) and traditional hydrogel lenses (etafilcon). The results showed that the amount and type of deposits on the lens are related to the lens material, with $\mathrm{Si}$-Hy lenses showing significantly more lipid deposits compared to ionic hydrogel material. There was also a difference in the size of the deposits between two Si-Hy lenses (fig. 1). The authors suggested that all of these differences can be related to the bulk material or surface treatment [16]. 


\section{FIGURE ( 1}

Degree of lipid deposition measured on Focus Night \& Day

(Iotrafilcon), PureVision (balafilcon), and Acuvue (etafilcon)

lenses. The degree of lipid deposition was significantly

influenced by both lens material $(p<0.001)$ and lipid class $(p<0.001)[16]$

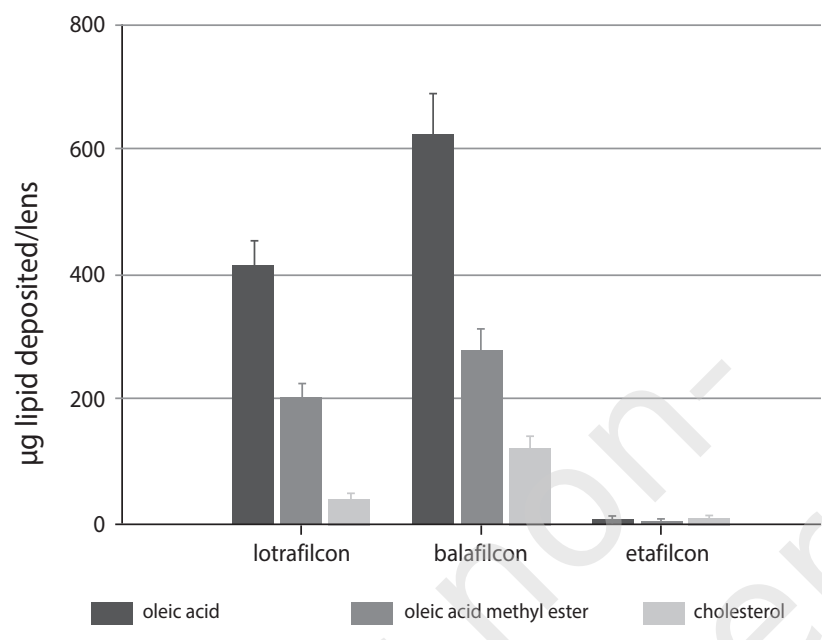

2004

Their next study was concentrated on an optimization of the procedure of the extracting lipid deposits from $\mathrm{Si}-\mathrm{Hy}$ lenses. Jones et al. were looking for the best extraction solution to remove lipids from PureVision (PV) and Focus Night\&Day (FND) lens materials. After examining both types of lenses (which were first placed in the Lipid Doping Solution) in four different extraction solutions, it turned out that the best solution was $2: 1$ chloroform: methanol which was capable of removing $>90 \%$ of deposited lipids from both PV and FND contact lens materials. They also demonstrated that PV lenses collect significantly more lipids compared to FND lenses [42].

\section{6}

A study which used high-performance liquid chromatography was published by Maziarz et al. The authors examined the sorption of oleic acid, oleic acid methyl ester, and cholesterol on Si-Hy contact lenses available at the time. They used two liquid chromatography methods (HPLC-1 developed by Bausch\&Lomb, and HPLC- 2 used by Jones et al., mentioned in the previous publication [16]) to analyze lens extracts from continuous-wear and daily-wear modalities from asymptomatic Si-Hy contact lens wearers. The most notable parameter difference between these two methods was the mobile phase composition and sample preparation technique. The study indicates that cholesterol is the most prevalently sorbed lipid in the studied contact lenses. They also suggested that the sorbed cholesterol may not be re- moved with cleaning solutions. What is more, they reported that the quantities of lipids sorbed to continuous-wear PureVision lenses were significantly different from those previously reported, and suggested that any hypothesis of $\mathrm{Si}-\mathrm{Hy}$ lenses based on previous lipid data should be reconsidered [43].

Jones et al. published an article on surface treatment, wetting and modulus of silicone hydrogels, in which they noted that lipid deposition onto Si-Hy lens materials is highly patient dependent. They recommended physical rubbing process to remove tenaciously bound lipids and denatured proteins that can be deposited on certain $\mathrm{Si}-\mathrm{Hy}$ lens materials in some patients [44].

\section{7}

Cheung et al. published a clinical comparison of Si-Hy (Acuvue Advance) and hydrogel (Acuvue) lenses. One of the evaluated parameters was the amount of deposits, which was assessed using a grading scale. There were no significant differences in the amount of deposits between the lenses, but Acuvue Advance was more often characterized by severe lipid deposits (third and fourth grade on the grading scale) (13.8\% during the first aftercare and $23.4 \%$ during the second aftercare visit). The authors noted that these deposits can be easily removed by rubbing the lens in the cleaning process [12].

The same year Lorentz et al. published a paper that describe the influence of lipids on the lens wetting angle. The study tested both $\mathrm{Si}$-Hy lenses (five $\mathrm{Si}$-Hy materials) and hydrogel lenses (four hydrogel materials). Lenses were incubated with two different lipid tear solutions (LTS) containing cholesterol, cholesteryl oleate, oleic acid, oleic acid methyl ester, and triolein, with "low" and "high" concentration of lipids, and compared with lenses soaked in phosphate buffered saline. The contact angle measurement (sessile drop method) showed that exposure to lipids may improve the wettability of certain Si-Hy and hydrogel materials, particularly of surface treated Si-Hy materials. Authors suggested that this may be the reason why in some individuals, the comfort of wearing lenses improves after a few hours or days after they have put on the lenses [45].

\section{8}

A study using atomic force microscopy (AFM) was published by Lira et al. They compared the surfaces of balafilcon A, lotrafilcon B (both surface-treated), and galyfilcon A (non-surface-treated) Si-Hy contact lenses, before and after having been worn by patients. They examined how and where proteins, lipids, and other contaminants change the contact lens surface. Balafilcon A and galyfilcon A showed a significant increase in surface roughness after being worn. The authors did not observed any significant changes in lotrafilcon B material. These results suggest 
that lens surface modifications can play a significant role in protecting from changes in roughness and allow for better clinical tolerance of the lenses [46].

Iwata et al. published a study on a new method of determining lipids adsorbed on Si-Hy lenses. This new method - gas chromatography/mass spectrometry (GC/MS) established in this study turned out to be effective in terms of repeatability, specificity, linearity, detection and quantitation limits, and percentage recovery. The authors suggested that GC/MS could be a useful method of analysis for evaluating lipids adsorbed on Si-Hy lenses. They evaluated in vitro lipids adsorbed on five $\mathrm{Si}-\mathrm{Hy}$ and one hydrogel lenses. The amount of lipids adsorbed on contact lenses were: lotrafilcon $\mathrm{A}=$ (near equal) lotrafilcon $\mathrm{B}=$ (near equal) etafilcon $\mathrm{A}<$ asmofilcon $\mathrm{A}<$ galyfilcon $\mathrm{A}=$ (near equal) balafilcon $\mathrm{A}$ with the value of 0.4 to $7.6 \mu \mathrm{g} /$ lens. The authors suggested that these differences in the amount of lipids adsorbed could depend on lipid composition and lens surface properties. This was also the first study to use other lipids than those found in the tear film, namely squalene that is a lipid secreted from the sebaceous gland of fingers/hands, which can be deposited on the lens after contact with the hands. The study has shown that squalene is easily deposited on lenses. On the other hand, cholesterol was shown to be easily absorbed into the lens [47].

The same year Carney et al. published an in vitro study on the adsorption of major tear film lipids to various $\mathrm{Si}$-Hy over time. Five Si-Hy and one hydrogel lenses were tested, with fluorescently tagged cholesterol ( $\mathrm{CH}$, non-polar lipid) and fluorescently tagged phosphatidylethanolamine (PE, polar lipid). After soaking the lenses in a lipid solution, researchers measured fluorescence and the corresponding lipid concentration was calculated from an appropriate standard curve. Study showed that the in vitro adsorption of $\mathrm{CH}$ was greater than that of PE for all lens types, including hydrogel. After 20 days of soaking in PE, the lotrafilcon polymers showed the lowest adsorption of all the $\mathrm{Si}$-Hy lenses tested at 0.4 and $1.5 \mu \mathrm{g} /$ lens, for lotrafilcon $\mathrm{A}$ and lotrafilcon B. Galyfilcon A and senofilcon A showed significantly higher PE adsorption at 5.1 and $4.9 \mu \mathrm{g} /$ lens, in comparison to all other Si-Hy lenses investigated. Senofilcon A and balafilcon $\mathrm{A}$ had the highest affinity for $\mathrm{CH}$ of all the lens types after 20 days, with adsorptions of 23.2 and $24.1 \mu \mathrm{g} / \mathrm{lens}$, respectively. Lotrafilcon B showed the lowest in vitro adsorption of $\mathrm{CH}$ of all lens types, at $3 \mu \mathrm{g} /$ lens [48]. Interestingly, the results of this study showed 10 times smaller amount of deposits on the same lenses than in the aforementioned study by Jones et al. [49]. The authors suggested that the differences between these in vitro and ex vivo studies may come from the protein-lipid interactions and drying and wetting cycles of a lens during wear. Another interesting conclusion was that the deposit characteristics of Si-Hy did not always behave according to the FDA lens material grouping of that time [48].

\section{9}

Two studies were published, discussing lens deposits and lens care products. Ngo et al. investigated the effect of proteins, lipids, and lens materials on the neutralization kinetics of one-step hydrogen peroxide disinfection. After adding protein (bovine serum albumin and lysozyme) and various lipids to the lens cases during the neutralization phase systems, they checked how this influenced the rate of neutralization. It turned out that neither protein nor lipid deposition nor lens material play a role in the speed of neutralization of peroxide-based systems [50].

Another study by Zhao et al. was focused on quantitatively detecting proteins and cholesterol extracted from worn $\mathrm{Si}-\mathrm{Hy}$ contact lenses. Researchers also wanted to determine the impact of various lens care solutions on deposit accumulation by using four lens types (lotrafilcon B, balafilcon A, galyfilcon A and senofilcon A) and four lens care solutions (ClearCare, Opti-Free Express, Opti-Free RepleniSH, AQuify), and thin layer chromatography for counting lipids and standard techniques for protein counting. The authors found that:

- Balafilcon A lenses exhibited the highest amount of cholesterol and total protein.

- AQuify was the most effective solution in reducing extracted deposits, especially proteins extracted from balafilcon A lenses.

- AQuify and Opti-Free RepleniSH solutions were most effective in reducing extracted cholesterol from senofilcon A and galyfilcon A lenses, respectively.

- The use of Opti-Free Express solution resulted in more extracted proteins from lotrafilcon B lenses than the use of other solutions.

- Lotrafilcon B, senofilcon A, and galyfilcon A lenses accumulated relatively low amount of proteins.

- Lotrafilcon B lenses accumulated the smallest amount of cholesterol deposits among all lenses tested regardless of the solution used.

The authors suggested that lens polymer (material) and surface characteristics may have a significant impact on the amount of deposits on the lenses and that lens polymer type, lens care solutions exhibit varying effectiveness in reducing protein and lipid accumulation [51].

\section{0}

Svitova et al. studied the influence of lysozyme and surfactant-containing multi-purpose lens care solutions (MPS) on interfacial rheology of lipids and mixed lipid-protein films. Although this study was not directly related to lens deposits, the results showed that thick multilayers 
of the ex vivo tear lipids (extracted from worn lotrafilcon A lenses) had exhibited lower surface tension than reported previously in the literature for meibomian lipids, and that lipid-lysozyme interaction altered the interfacial rheology of the ex vivo lipids. What is more, care solutions like, Opti-Free Express and Opti-Free RepleniSH changed the rheological properties of the mixed films to different extents [52].

In the same year, Pucker et al. published the results of a study aimed at developing an enzymatic method of quantifying cholesterol and cholesterol esters derived from contact lenses, both in vitro and ex vivo. They examined two types of materials, lotrafilcon B and galyfilcon A. The lenses underwent two separate 2: 1 chloroform-methanol extractions, then the amount of lipid deposits were measured using a cholesterol esterase enzymatic reaction. Both in vitro and ex vivo results showed that galyfilcon A material accumulated more lipids than lotrafilcon $\mathrm{B}$. In the conclusion, the authors suggested that this new method is an efficient and simple tool for measuring the total amount of cholesterol extracted from $\mathrm{Si}-\mathrm{Hy}$ contact lenses and, potentially, the meibum and/or tear film. They also pointed out that some $\mathrm{Si}$-Hy materials demonstrate more affinity for cholesterol and its esters than others [53].

The same authors published a second paper in the same year, whose aim was to understand various soft contact lens materials' ability to adsorb common tear lipids. They tested nine unworn polymers soaked in cholesterol oleate or phosphatidylcholine solutions for 1 or 14 days. The results obtained in this study are significantly different from those obtained by Iwata [47] and Carney [48]. Authors suggested that this may be a result of using: different methods, different non-polar lipids (cholesterol vs. cholesterol oleate), different polar lipids and different methods of analysis. In the conclusion, the authors informed that hydrogel and most of the Si-Hy lenses adsorb lipids relatively quickly (i.e. during the first day). Although there were some differences in the amount of lipids recovered from individual materials, it was not clear whether these differences could have any clinical significance [54].

Another study published in 2010 by Walter et al. investigated protein and lipid deposition characteristics of various $\mathrm{Si}-\mathrm{Hy}$ lenses. In terms of lipid depositions this study showed significantly less sorbed lipids in lotrafilcon A and B compared to other Si-Hy lenses. Authors suggested that not all $\mathrm{Si}$-Hy lenses have similar affinities for either protein or non-polar lipid (total cholesterol) sorption. Surface characteristics, such as ionicity, may play a significant role in the ability of these lens materials to resist deposition over a wear cycle [55].

Next paper from 2010, published by Saville et al., described examining the deposition of tear phospholipids and cholesterol onto worn contact lenses and the effect of lens mate- rial and lens care solution. Two Si-Hy lens materials (senofilcon $\mathrm{A}$ and balafilcon $\mathrm{A}$ ) and three types of solutions were used in the study. After extracting lipids with $2: 1$ (chloroform to methanol) solution and that extract having been washed with aqueous ammonium acetate, a lipid analysis was carried out using electrospray ionization tandem mass spectrometry. This study found that phospholipid deposits extracted from worn contact lenses show a molecular profile similar to that in tears. What is more, the lens composition significantly affected concentration of the representative polar and non-polar lipids deposited onto contact lenses. Differences were also shown in the efficacy of the removing phosphatidylcholine and sphingomyelin with lens care solutions [56].

The last reviewed study on the subject in this year, published by Zhao et al., described correlations between clinical responses during contact lens wear and the amount of protein or cholesterol extracted from lenses after wear. This study is a continuation of the same authors' 2009 publication [51], and it uses the same method to analyze deposits of protein and cholesterol on worn lenses. The researchers examined the correlation between the amount of protein and lipid deposits and such parameters as: corneal/conjunctival staining, lens front surface wetting and lens fitting tightness. They examined ex vivo four types of $\mathrm{Si}-\mathrm{Hy}$ lenses (senofilcon A, balafilcon A, galyfilcon A, lotrafilcon B), worn by patients daily on a biweekly or monthly basis, who used four types of lens solutions (ClearCare, Aquify, Opti-Free Express, Opti-Free RepleniSH). The obtained results showed that there are probably no physiologically relevant consequences of cholesterol depositing on Si-Hy lenses. However, the amount of proteins that deposit onto $\mathrm{Si}$-Hy lenses during wear may have more impact on lens performance on-eye. The correlations were generally weak and may still not indicate any relevant causative physiological response [57].

\section{1}

Walther et al. published a poster at British Contact Lens Association (BCLA) conference presenting the results of a study on the effect of in vitro lipid concentration on lipid deposition on Si-Hy and conventional hydrogel contact lens materials. After incubating four types of Si-Hy and two hydrogel lenses in artificial tear solution that contained major tear proteins, salts and either $0.5 \times, 1 \times$ or $2 \times$ typical human concentrations of selected lipids, they found that increasing concentrations of lipids resulted in an increase of the amount of cholesterol and phosphatidylcholine deposits. However, this rule did not apply to triolein. With these results the authors suggested that patients with elevated levels of certain lipids in their tear film, such as those which may occur in cases of meibomian gland dysfunction, could exhibit increased lipid deposition on their contact 
lenses, which may lead to compromised vision or end-of-day comfort [58].

Another study published at that time by Lorentz et al. focused on finding the physiologically-relevant artificial tear solution (ATS) containing a range of tear film components within a complex salt solution which would be relevant to measuring contact lens parameters and lipid deposition of a variety of contact lens materials after incubating in the ATS. The final step of this ATS development was tested for its ability to deposit lipids onto both a hydrogel and $\mathrm{Si}$-Hy contact lens materials. Cholesterol and phosphatidylcholine deposits on lenses of the omafilcon A (hydrogel) and balafilcon A ( $\mathrm{Si}-\mathrm{Hy})$ material were tested. The results showed that with ATS incubation balafilcon A lenses deposit significantly more cholesterol and phosphatidylcholine than omafilcon A lenses $(\mathrm{p}<0.05)$ and that removing lactoferrin and immunoglobulin $\mathrm{G}$ from the ATS can significantly decrease the amount of lipids deposited. In the conclusion, the authors stated that this solution (novel complex artificial tear solution especially designed for in-vial incubation of contact lens materials) was stable and did not adversely affect the physical parameters of the soft contact lenses incubated within it and showed that lipid deposition was responsive to changes in ATS composition [59].

In a study published by Heynen et al., the researchers quantified non-polar lipids deposited on senofilcon A Si-Hy contact lenses when disinfected with a no-rub onestep hydrogen peroxide system (ClearCare) and a care system preserved with Polyquad \& Aldox (OPTI-FREE RepleniSH). This ex vivo study found that among the symp- tomatic dry-eye soft lens wearers, a care system preserved with Polyquad and Aldox removed higher amounts of cholesterol oleate from senofilcon A contact lenses used for 2 weeks than a peroxide-based system [60].

Another study from 2011, published by Campbell, analyzed the impact of hand washing regimes on lipid transference to contact lenses. The authors compared a social hand wash, the Royal College of Nursing ( $\mathrm{RCN}$ ) hand wash and "no-wash regime" as the control group (only in transferring lipids from hands 'no CL used'), in ring lipid transfer from hands (thin-layer chromatography) and in transferring lipids to the CL (fluorescence spectroscopy). The study showed that RCN methods results in the lowest lipid transmission in both cases (fig. 2). Therefore, the authors suggested that a regime of hand washing for contact lens users should be standardized to help reduce potentially lipid transfer from hands [61].

Omali et al. studied the effect of cholesterol deposits on the adhesion of bacteria to $\mathrm{Si}$-Hy contact lenses. First, the researchers examined the lipid uptake from worn contact lenses (Pure Vision and Acuvue Oasys) ex vivo using thin-layer chromatography, and showed that these lenses absorbed cholesterol. Then they soaked unworn lenses in cholesterol and the number of Pseudomonas aeruginosa strains or Staphylococcus aureus strains that adhered to the lenses were measured. Cholesterol was tested for effects on bacterial growth by incubating bacteria in a solution containing cholesterol. The results suggested that this lipid does not appear to modulate bacterial adhesion to a lens surface [62].

\section{FIGURE $(2$}

Fluorescence intensity (FI) of dermal-lipids on contact lenses after no wash, social wash and RCN (Royal College of Nursing) wash [61].

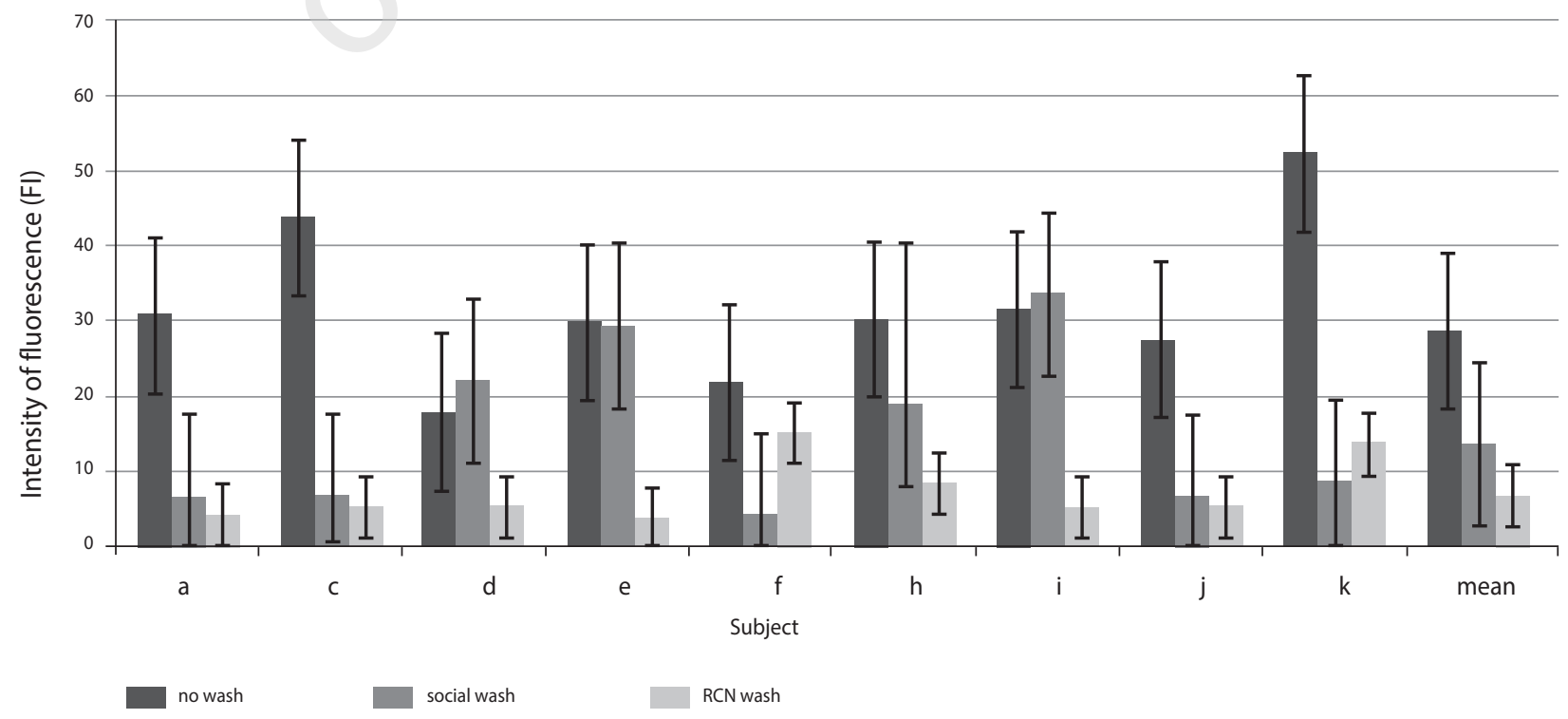


The aim of a study published by Pitt et al. was to check the possibility of loading a phospholipid onto contact lenses for a controlled release onto the eye. The lens was loaded with $33 \mu \mathrm{g}$ of DMPC (1,2-dimyristoyl-sn-glycero-3-phosphocholine). An average of nearly $1 \mu \mathrm{g}$ of DMPC was eluted into ATF (artificial tear solution) within the first $10 \mathrm{~h}$. What is more, the elution was about five times faster in ATF than in water. Authors suggested that this type of lens technology may have the potential to deliver phospholipids to help address contact lens-related dryness through lipid layer stabilization [63].

\section{2}

Pucker et al. published a study on imaging lipid deposits on Si-Hy lenses. They examined the ability of Nile Red and Oil Red O stain to detect tear film lipids deposited on Si-Hy lenses. The authors examined both in vitro and ex vivo lenses, and found that Nile Red and Oil Red $\mathrm{O}$ are both able to detect lipids on soft lenses in both cases. Oil Red O was described as a better stain for $\mathrm{Si}$-Hy lenses as it offers a higher signal to noise ratio [64].

Vishnubhatla et al. published a study on the influence of wear, squalene and wax on the evaporation rates through contact lenses (ERTCL). They found that the ERTCL through worn balafilcon A contact lenses were about $20 \%$ faster than for buffer saline alone and that squalene inhibited the ERTCL by over $60 \%$ [65].

As a continuation of previous studies from 2011 [62] Omali et al. published a study about the effect of phospholipid deposits on bacteria adhesion to contact lenses. First, the authors examined worn lenses to check the amount of phospholipid deposits. As the next step, unworn lenses were soaked in phospholipids and exposed to Pseudomonas aeruginosa and Staphylococcus aureus. After an $18 \mathrm{~h}$ period of incubation, the number of $P$. aeruginosa or S. aureus bacteria that adhered to the lenses were measured. The results showed that phospholipids adsorbed onto/absorbed by contact lenses during wear, however, major types of phospholipids adsorbed to lenses do not alter bacterial adhesion or growth [66].

Another study published in 2012 described the impact of lactoferrin and lipids on the kinetic deposition of lysozyme on $\mathrm{Si}$-Hy and conventional hydrogel lenses, using a complex artificial tear solution (ATS). Researchers examined two Si-Hy (lotrafilcon B and senofilcon A) and two hydrogel (etafilcon A and omafilcon A) lens materials with four different solutions:

- a complex ATS consisting of various salts, lipids, proteins, and mucins

- an ATS without lactoferrin

- an ATS without lipids

- an ATS without lactoferrin and lipids.
The results showed that the ATS containing lactoferrin and lipids affects lysozyme deposition on both $\mathrm{Si}-\mathrm{Hy}$ and conventional hydrogel contact lenses [67].

The development of in vitro experiments was focused on achieving conditions as close as possible to in vivo. A step in achieving the aim was taken by Lorentz et al. by incorporating a model blink cell device in examination of lipid deposits. The published study was aimed to analyze the impact of intermittent air exposure on in vitro deposition of two radioactive lipids on various contact lens (CL) materials. There were two stages of the study, short term ( 1 day $\times 10$ h of continuous cycles in and out of ATS) and long term ( 6 days $\times 14 \mathrm{~h}$ continuous cycles in and out of ATS and $10 \mathrm{~h}$ submerged in ATS). In the first stage, the authors examined six different CL materials (balafilcon A, lotrafilcon B, comfilcon A, senofilcon A, etafilcon A, and omafilcon A) which were mounted on the model blink cell pistons, which cycled the lenses in and out of a complex artificial tear solution (ATS) that contained a trace amount of ${ }^{14} \mathrm{C}$-cholesterol or ${ }^{14} \mathrm{C}$-phosphatidylcholine. In these cases, the exposure to air resulted in increased amounts of cholesterol deposited, 1.6 to 4.3-fold for omafilcon A, balafilcon A, comfilcon A, and senofilcon A compared with submerged lenses, however, no differences in deposition were observed for etafilcon A and lotrafilcon B. For phosphatidylcholine, the all air-exposed lenses had increased the amount of deposition. These deposits were statistically higher by 1.1 to 1.6 times for omafilcon A, comfilcon A, lotrafilcon B, and senofilcon A, but not statistically different for etafilcon A or balafilcon A. Long term incubations for 6 days were tested with two Si-Hy (lotrafilcon B and balafilcon A) materials incubated in ${ }^{14} \mathrm{C}$-cholesterol ATS. The air-exposed CLs were cycled for $14 \mathrm{~h}$, then submerged for $10 \mathrm{~h}$ each day. This time results showed statistically significant increases in cholesterol deposition for both air-exposed lens materials with the increase in deposition $1.8 \times$ and $2.8 \times$, respectively. In the conclusion, the authors suggested that lipid deposition profiles are CL material dependent and that intermittent air exposure can influence the amount of lipids deposited [68].

Lorentz et al. published another study in 2012. The purpose of their work was to analyze the influence of various tear film components on in vitro deposition of two lipids (cholesterol and phosphatidylcholine) on contact lens materials. They examined two Si-Hy (balafilcon A and senofilcon A) and one hydrogel (etafilcon A) lens materials with four solutions:

- an artificial tear solution containing lipids and proteins

- a protein tear solution containing proteins and the lipid of interest

- a lipid tear solution containing lipids and no proteins

- a single lipid tear solution containing the lipid of interest only. 
Each incubation solution contained one of the two radiolabeled lipids: ${ }^{14} \mathrm{C}$-cholesterol $(\mathrm{C})$ or ${ }^{14} \mathrm{C}$-phosphatidylcholine (PC). Lenses were incubated for 3 and 14 days. The results showed that that $\mathrm{C}$ and $\mathrm{PC}$ deposition accumulates over time and that $\mathrm{Si}-\mathrm{Hy}$ materials deposit more lipid than group IV conventional hydrogel materials. However, a more significant conclusion was that the deposition of $\mathrm{C}$ and PC is affected by the composition of the incubation solution and that in vitro models need to use more physiologically relevant incubation solutions that mimic the natural tear film if in vitro data is to be extrapolated to the in vivo situation [69].

As a continuation of study from 2011 [63], Pitt et al. published another paper in 2012 on the transport and release of phospholipids from Si-Hy contact lenses. The results showed that the amount of DMPC loaded into a lens is a linear function of time of exposure to the DMPC/propanol solution (used to load DMPC onto lens). The initial rate of elution into ATF appeared to be diffusion-controlled for at least $10 \mathrm{~h}$ and is proportional to the amount of DMPC loaded. What is more, the elution rate decreases as the DMPC concentration in the ATF increases. In the conclusion, the researchers stated that the ease of loading and controllable release of DMPC from Si-Hy presents the possibility of using such lenses to counter eye discomfort caused by inherently low levels of phospholipids in tears [70].

The last reviewed study from 2012 aimed to assess the efficiency of hydrogen peroxide solutions in removing lipids from various contact lens materials by using an in vitro model of lipid deposition. Authors examined two Si-Hy (balafilcon A and senofilcon A) and one hydrogel (etafilcon A) lens materials which were incubated for $8 \mathrm{~h}$ in an ATS containing a mixture of lipids, proteins, mucin and either ${ }^{(14)} \mathrm{C}$-cholesterol or ${ }^{\left({ }^{14}\right)} \mathrm{C}$-phosphatidylcholine. Following the incubation, the lenses were removed, rinsed, and placed for $16 \mathrm{~h}$ in either a surfactant-containing a peroxide solution (ClearCare ${ }^{\odot}$ ), a peroxide solution devoid of a surfactant (AOSept ${ }^{\oplus}$ ) or stored without solution (control). After 1 week of repeating the process each day the amount of lipids deposited were calculated based on standard calibration curves. The results showed that ClearCare hydrogen peroxide disinfection solution containing Pluronic 17R4 removed the most lipids from lenses when compared with the non-surfactant containing AOSept or the control, for both lipids and all lens materials. However, the authors noticed that the differences found were quite small at times and whether these differences are clinically significant are yet to be determined [71].

\section{3}

Brown et al. published a study that described a method of characterizing lipid deposits directly from worn contact lenses utilizing a liquid extraction surface analysis coupled with mass spectrometry (LESA-MS/MS). The presented technique effected a facile and reproducible extraction of lipids from the contact lens surfaces and identified lipid molecular species representing all major classes present in human tear film. The authors stated that the LESA-MS/MS is a rapid and comprehensive technique for characterizing lipid-related biofouling on polymer surfaces [72].

As a continuation of the 2012 study [67], Ng et al. published in 2013 an article about the impact of tear film components on the conformational state of lysozyme deposited on contact lenses. They examined two Si-Hy (lotrafilcon B and senofilcon A) and two hydrogel (etafilcon A and omafilcon A) lens materials, which were incubated in four different solutions: ATS, ATS without lactoferrin, ATS without lipids, ATS without lactoferrin and lipids.

At various time points over a 28-day period, the percentage of active lysozyme per lens was determined using a fluorescence activity assay and an enzyme-linked immunosorbent assay (ELISA). The results showed that lactoferrin and lipids have an impact on the denaturation of lysozyme deposited onto $\mathrm{Si}$-Hy contact lenses, while conventional hydrogel lenses were unaffected [73].

In another study published by Pitt et al. they searched for a best method of quantitating phospholipid and cholesterol sorption on $\mathrm{Si}-\mathrm{Hy}$ lenses using radiolabeled cholesterol and phosphatidylcholine that were sorbed on lenses from ATF. The results showed that a triple extraction technique using $\mathrm{n}$-propanol gives the most reliable results. What is more, the comparison of sorption on $\mathrm{Si}$-Hy lenses showed that balafilcon A and senofilcon A lenses sorb similar amounts, while lotrafilcon B lenses sorb comparatively less lipids [74]. Svitova et al. published a study investigating racial variations in interfacial behavior of lipids extracted from worn soft contact lenses. Asymptomatic Asian and Caucasian subjects wore Si-Hy lenses (lotrafilcon A) continuously for 1 month. Then, lipids from lenses were extracted and deposited on an air bubble immersed in a model tear electrolytes (MTE) solution to form $100 \pm 20 \mathrm{~nm}$-thick films. Surface pressure was recorded during slow expansion/ contraction cycles to evaluate compressibility and hysteresis of lipid films. The films were also subjected to fast stepstrain dilatations at temperatures $22-45^{\circ} \mathrm{C}$ to assess their viscoelastic properties. The researchers found that higher elastic modulus of Caucasian lipids and elasticity threshold at certain deformations indicate stronger intermolecular interactions and structure in comparison with more viscous Asian lipids. Moreover, they suggested that the differences in interfacial behavior between Asian and Caucasian lipids may be associated with the differences in their chemical compositions [75].

In a comprehensive study of the factors that influence in vitro cholesterol deposition on contact lenses, published in 2013 by Walther et al. information about the impact of 
incubation time, lipid concentration, and solution replenishment on Si-Hy and conventional hydrogel contact lens cholesterol deposition in vitro can be found. The researchers examined four Si-Hy (senofilcon A, lotrafilcon B, comfilcon A, balafilcon A) and two hydrogel (etafilcon A and omafilcon A) contact lenses incubated ATS, that contained major tear film proteins, lipids, salts, salts, and a trace amount of radioactive ${ }^{14} \mathrm{C}$-cholesterol, for various incubation times $(1,3,7,14$, or 28 days), with three concentrations of lipids $(0.5 \times, 1 \times, 2 \times$ tear film concentration) and with or without solution replenishment to assess the impact of each variable on cholesterol deposition. The results showed that all three factors influence in vitro cholesterol deposition. Si-Hy lenses deposit significantly more cholesterol than hydrogel lens materials, and the amount of lipids deposited depends on the contact lens material, length of incubation, concentration of lipids in the ATS, and the replenishment of ATS [76].

\section{4}

Walther et al. published a study to compare ex vivo cholesterol deposition on commercially available Si-Hy contact lenses. They examined seven Si-Hylens materials (balafilcon A, comfilcon A, enfilcon A, enhanced-lotrafilcon A, enhanced-lotrafilcon B, galyfilcon A and senofilcon A). Cholesterol sorption was assessed in 140 previously worn $\mathrm{Si}$-Hy lenses from eight clinical trials conducted in the United States and Australia between 2006 and 2008. Patients wore the lenses for 2-4 weeks. The results demonstrated that enhanced-lotrafilcon A and enhanced-lotrafilcon B contact lenses showed significantly lower median total cholesterol sorption than all other types of tested lenses. The authors suggested that cholesterol deposition in $\mathrm{Si}-\mathrm{Hy}$ contact lenses depends on the lens material [77].

Panaser et al. published a study on the evidence of lipid degradation during overnight contact lens wear. Using gas chromatography and mass spectrometry they examined (ex vivo) two lens materials (balafilcon A or lotrafilcon A) which were worn on a daily or continuous wear schedule for 30 and 7 days. Obtained results showed that:

- Unsaturated fatty acids are degraded during sleep in contact lenses.

- Degradation occurred independently of lens material or subject-to-subject variability in lipid deposition.

- The consequence of lipid degradation is the production of oxidative products, which may be linked with contact lens discomfort.

What's more, they found differences between balafilcon A and lotrafilcon A lenses, primarily relating to higher levels of gross lipids on balafilcon A lenses [78].

The same year Maissa et al. published a study comparing lipid spoliation on $\mathrm{Si}$-Hy (balafilcon A) and hydrogel (etafilcon A) lenses. They examined lenses collected from patients who wore them for $10 \mathrm{~h}$ of single use (DD) and 7 days of extended wear (EW). The authors asked two laboratories to analyze lipids: Alcon Laboratories (right lens total uptake) and OTG Research \& Consultancy (left lens total uptake and individual lipid classes). The results showed that total lipid uptake was highly material dependent. Measurements showed a greater uptake of lipids by the $\mathrm{Si}$-Hy than the hydrogel material. Total lipid uptake was greater after 7 days of EW compared with $10 \mathrm{~h}$ of DD. The kind of lipids recovered from the contact lenses was also significantly different between the two materials: more nonpolar lipids were recovered from balafilcon A, whereas the distribution of lipid families was more balanced between non-polar and polar lipids for etafilcon A. Greater differentiation between materials was possible after 7 days of EW for each material. The lipid uptake profile was similar for DD and EW, indicating the material being a more important factor than wear modality [79].

Another study published in 2014 compared lens solutions ability to remove tear components. Cheung et al. examined three $\mathrm{Si}-\mathrm{Hy}$ lens materials (balafilcon A [BA], lotrafilcon $B[L B]$, and senofilcon $A[S A])$, which were incubated for 1 week in ATS containing representative lipids, proteins, and salts from the tear film. AFM was used to resolve each lens before and after being cleaned overnight in hydrogen peroxide based system (HPS) or polyhexamethylene biguanide containing MPS. In the conclusion, the authors stated that unique variations in ATS deposition patterns were seen between lenses with AFM, and that the application of both HPS and MPS removed most of visible surface deposits. However, in terms of lipid deposition, the researchers found that the results for balafilcon A and senofilcon A were not consistent with the studies on lipid adsorption, because BA and SA have been shown to deposit relatively high levels of lipids [48, 69, 74]. One possible explanation for this discrepancy is that lipids, and proteins to a lesser extent, are being absorbed into the matrix of BA, whereas they are adsorbed and visible on the surface of SA [80].

Tam et al. published two studies in 2014 on lipid deposition and lens care solutions. In the first one, they examined the sorption and desorption of radio-labeled dipalmitoylphosphatidylcholine (DPPC) and cholesterol $(\mathrm{CH})$ on five types of commercial contact lenses. First, the lenses were soaked in vitro in an ATS for $16 \mathrm{~h}$. Then the effects of borate buffered saline and two commercial MPSs on reducing the lipid (DPPC and $\mathrm{CH}$ ) sorption and increasing the lipid removal were examined. The results showed that $\mathrm{Si}-\mathrm{Hy}$ lenses accumulate more lipids than polymacon (hydrogel) material. Pre-soaking the Si-Hy lenses for $16 \mathrm{~h}$ in MPSs reduced the DPPC sorption by up to $13 \%$ and the $\mathrm{CH}$ sorption by up to $11 \%$, but these reductions were not statistically significant. The results for lipid removal showed that the composition of the eluting solution, whether BBS or an 
MPS, appears to have only a small influence on removing lipids from $\mathrm{Si}$-Hy lenses [81].

The second study from Tam et al. 2014, analyzed the efficiency of lens care solutions in preventing and removing lipid deposits from contact lenses. This time the authors took also the rubbing process into consideration. Once again, MPS did not show efficiency in preventing and removing lipids from contact lenses. In the conclusion, the authors stated that obtained data suggest that MPSs do not appreciably alter lipid sorption. Rubbing the lenses removes a small amount of sorbed lipids. Yet, authors recommend that MPSs should be used as they may disinfect Si-Hy lenses and clean their surfaces from large particles [82].

\section{5}

In a study published by Bhamla et al. they found that modifying the Si-Hy surface with simple lipids, such as DPPC and cholesterol, increases the hydrophilicity, which consequently inhibits dewetting, whereas meibum behaves conversely. Additionally, they observed that DPPC and meibum remain closer to the $\mathrm{Si}-\mathrm{Hy}$ surface than cholesterol, which diffuses further into the porous Si-Hy matrix [83]. Hagedorn et al. published a study on assessing contact lens deposits and human meibum extracts using AFM and the Langmuir-Blodgett monolayer. In the part dedicated to contact lens deposits they examined ex vivo two $\mathrm{Si}-\mathrm{Hy}$ (etafilcon A and balafilcon A) materials worn in two phases by the same subjects. The results for CL with AFM suggest that the non-MGD participants wore lenses containing a more uniform spread of smaller lipid deposits with higher roughness values than the MGD lenses. Researchers speculated that this may occur because the tear film structure of a person without MGD is relatively stable and ordered, enabling fewer lipid and protein interactions with the CL once inserted. They also found that depth analysis of balafilcon A pore suggests that lipids may accumulate within the pores of the material when worn. What is more, this phenomenon was more significant in the lenses worn by MGD patients, as these lenses had shallower pores when compared to non-MGD patient lenses which had deep pores. In the conclusion, the authors stated that lipid deposition on CL differs in terms of amount and pattern between non-MGD and MGD groups and in terms of types of lipids between etafilcon A and balafilcon A lens materials. Lipids from patients with MGD are more disordered and deposit irregularly on CL, whereas lipids from non-MGD patients are relatively ordered and deposit relatively uniformly on CL [84].

As a continuation of the studies from 2011 and $2012[63,70]$ Pitt et al. published another study on extending the elution of phospholipid from Si-Hy contact lenses. The researchers examined an experimental lens simulating 30 days of diurnal use with overnight cleaning. The amount of released radio-labeled DMPC was measured. The results showed that the elution of DMPC into ATS was greater on the first day, followed by a fairly constant amount of elution each day thereafter. The study also found that the type of cleaning system had a statistically significant effect on the elution rate during daily exposure to ATS. The rate of elution into cleaning solutions did not show any enhanced elution on the first day; there was a fairly constant elution rate [85]. In the aforementioned studies on lipid deposits on $\mathrm{Si}-\mathrm{Hy}$ lenses, the focus was on reusable lenses, however another study from 2015, published by Walther et al. examined in vitro cholesterol deposition on daily disposable contact lens materials. The authors analyzed three Si-Hy (somofilcon A, delefilcon A, and narafilcon A) and four hydrogel (etafilcon A, nesofilcon A, ocufilcon A, and nelfilcon A) lens materials. After incubating in ATS with radioactive ${ }^{14} \mathrm{C}$-cholesterol, and extracting, the extracts were analyzed in a beta radiation counter. The first finding was that cholesterol deposited statistically significantly more on Si-Hy lenses than hydrogel (with some exceptions). There were also differences between Si-Hy lenses. The authors found also that the accumulation of cholesterol was shown to be continuous throughout the $16 \mathrm{~h}$ of incubation, without reaching a plateau (as shown in figure 3 ). The authors suggested that those facts could have implications for wearers who have higher levels of lipids in their tears and are fitted with Si-Hy DD materials [86].

Further work was carried out to get the in vitro test environment as close to in vivo as possible. Peng et al. published a study on an in vitro model-blink cell that reproduces the mechanism of in vivo fouling of soft contact lenses. With this novel method of incubating lenses in ATS the authors examined 1 hydrogel (etafilcon A) and five Si-Hy (galyfilcon A, lotrafilcon A, lotrafilcon B, balafilcon A, comfilcon A) materials. Deposits were analyzed using optical microscopy, laser ablation electrospray ionization mass spectrometry, and two-photon fluorescence confocal scanning laser microscopy. As a result, they found that targeted lipids (cholesterol) and proteins (albumin from bovine serum) were identified in the discrete surface deposits. Both lipids and proteins occur simultaneously in the surface deposits and overlap with the white spots observed using optical microscopy. An examination using fluorescence confocal scanning laser microscopy showed that lipids and proteins penetrate into the bulk of tested Si-Hy lenses, likely attributed to the bi-continuous microstructure of oleophilic silicone and hydrophilic polymer phases of the lens [87].

The last reviewed study from 2015, published by Silva et al., aimed to evaluate the effect of albumin and cholesterol on the biotribological behavior of hydrogels for contact lenses. Researchers examined two materials: hydroxyethylmethacrylate based hydrogel, HEMA/PVP, and a silicone-based one, TRIS/NVP/HEMA. Tribology examinations showed 


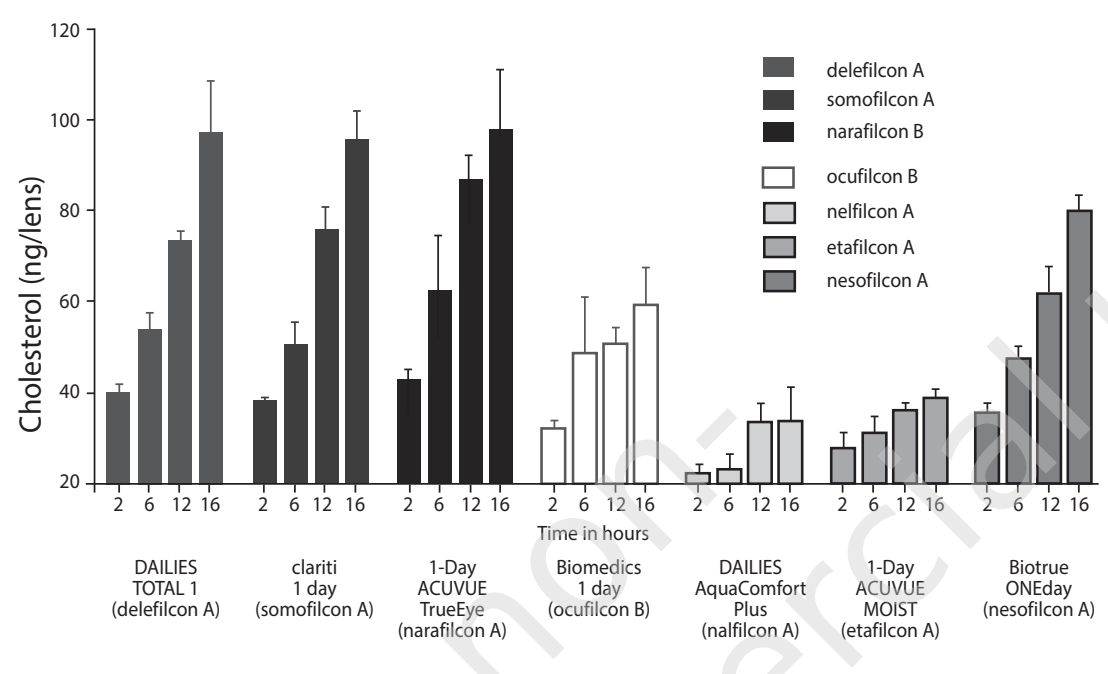

a significant increase in the friction coefficient $(\mu)$ for HEMA/PVP when the lubricant contains cholesterol and for TRIS/NVP/HEMA when it contains albumin. The authors stated that the results contribute to understanding the influence of lacrimal fluid composition on the tribological behavior of CLs materials, and can lead to improvement of the selection and optimization of these devices [88].

\section{6}

Bassyouni et al. published a study on the possibility of using cinnamon oil to disinfect contact lenses. With a good result of minimum inhibitory concentrations (MIC) of tobramycin and cinnamon oil against 19 bacterial strains authors stated that cinnamon oil has a promising antimicrobial effect and it could be a probable candidate for contact lens disinfection. There is no information in the study about possible oil deposits on contact lenses and the potential for reducing the wettability of lens surfaces [89].

Another study from 2016 aimed to examine the effects of two weeks of regular phospholipid liposomal spray application on lipid layer grade, tear film stability, subjective comfort, visual acuity, and lipid deposition in Si-Hy contact lens wearers. Phospholipid supplementation for contact lenses is considered controversial due to possible lipid deposits in the lens materials (especially $\mathrm{Si}-\mathrm{Hy}$ ). However, the results obtained in this study showed that using a phospholipid liposomal spray increased tear film stability, lipid layer thickness and subjective comfort in Si-Hy contact lens wearers, without adversely affecting visual acuity or contact lens surface lipid deposition [90].

\section{7}

Schuett et al. published a work on an experimental model to study the impact of lipid oxidation on contact lens deposition in vitro. The authors examined two Si-Hy materials (balafilcon A and senofilcon A) which were incubated with fatty acids laced with radioactive tracer oxidized to varying degrees, and the amount of lipid deposition was measured using unoxidized lipid samples as controls. The results showed that saturated fatty acids are not oxidized, the monounsaturated oleic acid produced peroxides while poly-unsaturated lipids initially produced peroxides and then fragmented into reactive aldehydes. The authors stated that they provided a method for inducing and controlling lipid oxidation so that the effect of lipid oxidation on contact lens binding could be compared [91].

The second study from 2017 determined the effect of lens care system combinations on levels of total lipid, cholesterol, and cholesteryl esters, extracted from contact lenses (CLs), and was published by Omali et al. The authors examined ex vivo 791 lenses (three different materials: two Si-Hy (galyfilcon A, senofilcon A) and one hydrogel (etafilcon A), which were worn for 10-14 days by subjects and cleaned using one of four solutions (Biotrue, ClearCare, OPTI-FREE PureMoist, and RevitaLens Ocutec). Then, lens were analyzed to determine the amount of cholesterol, cholesteryl esters, and total lipids. However, the results did not demonstrate conclusively that any of the solution/CL combinations were superior to any of the other combinations when the amounts of lipid deposition were compared among the tested lenses [92]. 


\section{8}

Walther et al. examined fluorescently tagged cholesterol uptake and penetration in daily disposable contact lenses. The authors studied three Si-Hy (delefilcon A, somofilcon A, narafilcon A) and four hydrogel (etafilcon A, ocufilcon B, nesofilcon A, nelfilcon A) materials. The incubation in ATS of mentioned lens was performed on in vitro eye-blink platform designed to simulate physiologic tear flow $(2 \mathrm{ml} / 24 \mathrm{~h}$ ), tear volume and "simulated" blinking. Then, the lenses were analyzed using laser scanning confocal microscopy. The results showed that compared with traditional incubation in ATS (where NBD-cholesterol uptake occurred equally on both sides of all lens materials), in the eye-blink model cholesterol penetration was observed primarily on the anterior surface of the lenses (fig. 4). In the conclusion, the authors suggested that comparing to the eye-blink platform method the traditional "in-vial" incubation method exposes CLs to an excessively high amount of ATS, which results in an overestimation of cholesterol deposition. They also stated that this new model, which incorporates important ocular factors, such as intermittent air exposure, small tear volume, and physiological tear flow between blinks, provides a more natural environment for the in vitro lens incubation [93].

\section{9}

Walther et al. published a study on the efficacy of contact lens care solutions in removing cholesterol deposits from Si-Hy contact lenses. By examining five Si-Hy lens materials: (senofilcon A, comfilcon A, balafilcon A, lotrafilcon A, and lotrafilcon B) previously incubated in ATS containing radiolabeled cholesterol, with a saline solution and five MPSs (Biotrue MPS, renu fresh, Blink RevitaLens MPS, OPTI-FREE PUREMOIST MPDS, SoloCare Aqua), they found that:

- Balafilcon A and senofilcon A lens materials showed the highest amounts of accumulated cholesterol.

- Lotrafilcon A and lotrafilcon B deposited the lowest amounts.

- For all lens materials, MPS preserved with POLYQUAD/ ALDOX (OPTI-FREE PUREMOIST MPDS) removed more deposited cholesterol than any other test solution.

The results of total cholesterol uptake five lens materials combined with saline solution and five MPSs are shown in figure 5. In the conclusion, the authors stated that cholesterol-removal efficacy varies depending on the combination of lens material and solution, and that only one MPS showed a statistically significant reduction of cholesterol deposits for only two of the five tested lens materials [94].

\section{FIGURE ( 4}

Confocal images showing cross-sections of etafilcon A, nelfilcon A, nesofilcon A,

ocufilcon, delefilcon $A$, somofilcon A, narafilcon $A$ after incubation with NBD-

-cholesterol in the vial and OcuFlow model after $4 \mathrm{~h}(\mathrm{~A})$ and $12 \mathrm{~h}$ (B) [93].

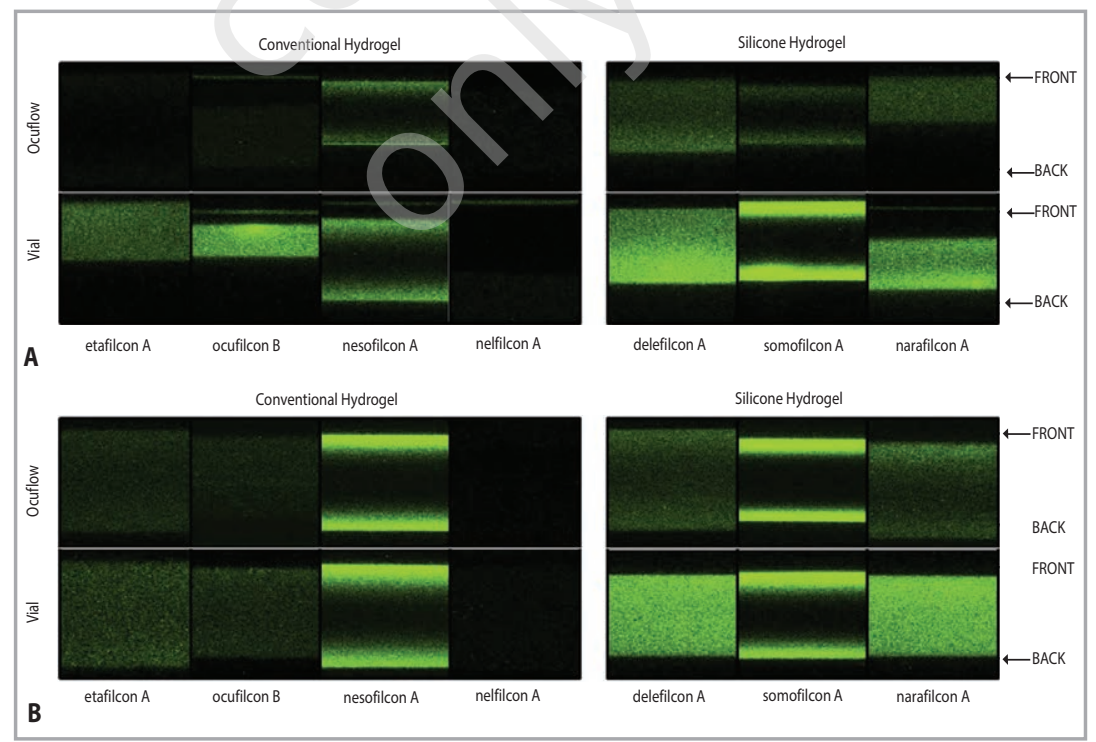




\section{FIGURE ( 5}

The mean ( \pm SD) total cholesterol uptake on various lens material when soaked and cleaned in saline and five different MPSs after 7 days of incubation in a complex tear solution containing radiolabeled ${ }^{14} \mathrm{C}$-cholesterol ( ${ }^{*} p=0.05$ for cleaned vs. uncleaned lenses). MPSS, multipurpose solutions [94].

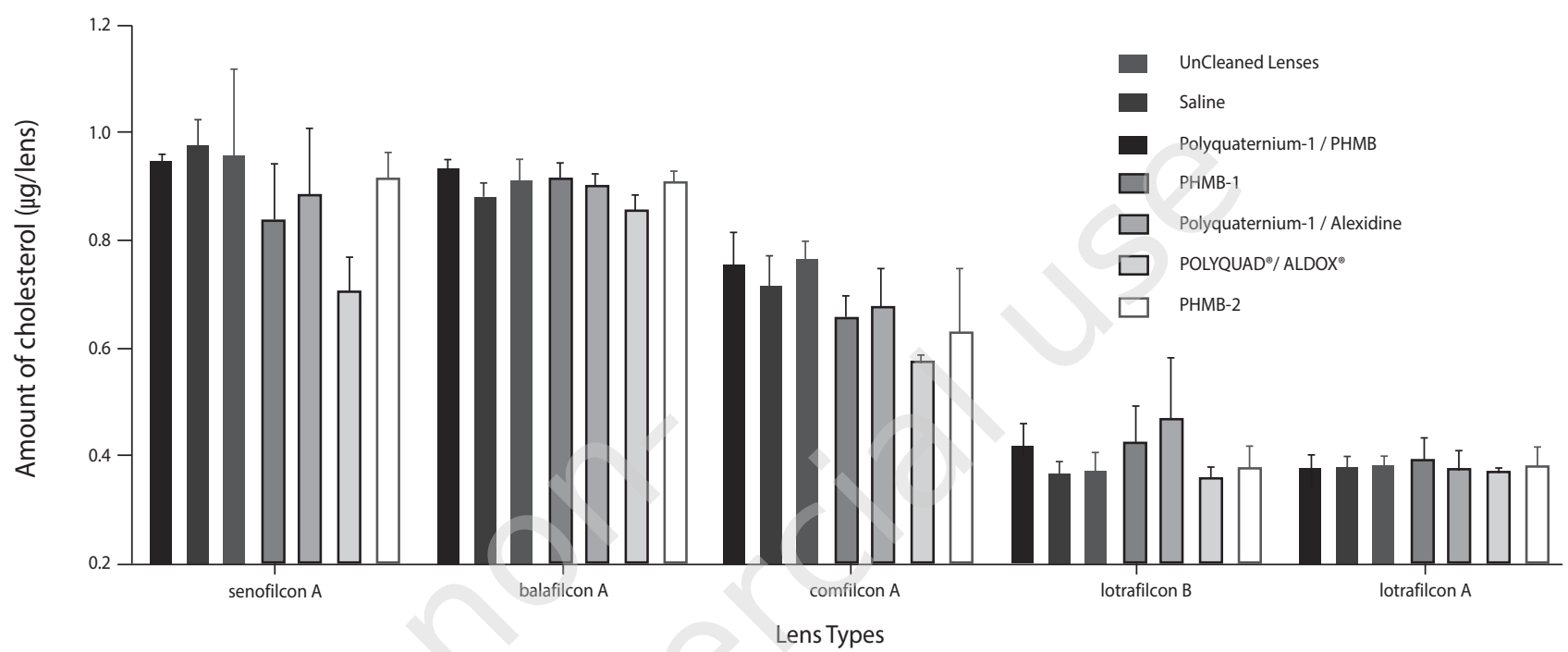

\section{0}

Luensmann et al. published a study investigating kinetic lipid uptake in $\mathrm{Si}-\mathrm{Hy}$ lenses. The authors, simulating monthly use, examined four Si-Hy (lotrafilcon B, senofilcon C, comfilcon A, samfilcon A) lens materials with incubation in three different solutions: ATS containing ${ }^{14} \mathrm{C}$-labeled phosphatidylcholine, ATS containing ${ }^{14} \mathrm{C}$-cholesteryl oleate, and ATS containing four ${ }^{14} \mathrm{C}$-radiolabeled lipids (phosphatidylcholine, phosphatidylethanolamine, cholesteryl oleate and cholesterol). Results showed that for all studied lens types the total lipid amounts increased over time. The highest amount of total lipids was found on senofilcon $C$ lens, cholesteryl oleate on lotrafilcon B and phosphatidylethanolamine on senofilcon $C$. The researchers stated that the amount of both polar and non-polar lipid depositions on monthly replacement $\mathrm{Si}$-Hy lenses increased over 4 weeks, with significant differences being seen between lens materials [95].

Another study from 2020, published by Qiao et al., evaluated the location of cholesteryl ester deposits in monthly $\mathrm{Si}$-Hy lenses under conditions that mimic a daily wear regimen over 1, 14 and 30 days. The authors examined four Si-Hy lenses (senofilcon C, lotrafilcon B, comfilcon A and samfilcon A) incubated in ATS including fluorescently tagged cholesteryl ester (CE-NBD) for $16 \mathrm{~h}$ every day and $8 \mathrm{~h}$ in a MPDS solution (OPTI-FREE ${ }^{\oplus}$ PureMoist $^{\circledR}$ ), with confocal laser scanning microscopy. The results showed that the distribution of the non-polar lipid CE-NBD varied depending on the lens material. Some lens materials (lotra- filcon B and samfilcon A) deposited the lipid primarily on the surface after $16 \mathrm{~h}$ of exposure at day 1 and day 14 . However, all materials exhibited a homogenous distribution after one month (fig. 6) [96].

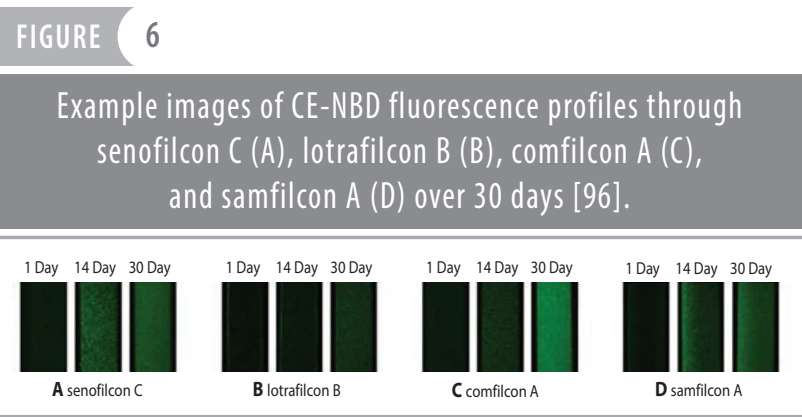

The purpose of the study published by Shows et al. was to compare the extracted cholesterol of lotrafilcon B lenses packaged in and cared for with ethylene oxide-butylene oxide (EOBO)-containing lens care solutions with the extracted cholesterol of habitual Si-Hy lenses cared for with MPS not containing EOBO. In this study the author first examined cholesterol extracted from habitual worn lenses (senofilcon C, senofilcon A, comfilcon A, and samfilcon A) which were used with a non-EOBO (polyoxyethylene-polyoxybutylene) MPS, and next they examined cholesterol extracted from lotrafilcon $\mathrm{B}$ lens used with EOBO-containing lens care solutions, OPTI-FREE PUREMOIST or CLEAR 
CARE PLUS with HydraGlyde (in Europe AOSEPT plus with HydraGlyde) used by the same subjects. The results showed that cholesterol sorption was significantly lower in wearers of lotrafilcon B lenses cared for with EOBO-containing lens care solutions than in users of habitual $\mathrm{Si}-\mathrm{Hy}$ lenses cared for with non-EOBO MPS [97].

A study by Omali et al. quantified lipid deposition on CLs in a group adapted of symptomatic and asymptomatic CL wearers. Confirmed symptomatic and asymptomatic participants ( 50 in total; 25 per group) have been tested in these phases. All participants were refitted with a 2-weekly replacement $\mathrm{Si}-\mathrm{Hy}$ lens (senofilcon $\mathrm{A}$ ) and issued a polyquaternium-based care solution (OPTI-FREE ${ }^{\bullet}$ RepleniSH ${ }^{\circ}$ ). Participants wore a new pair of lenses for the following 2 weeks. The results showed that the asymptomatic group deposited a significantly greater amount of lipids on their CLs. Although measured lipid levels are considered low to trigger any observable clinical deposition, they may influence other clinical outcomes, particularly on comfort [98].

\section{DISCUSSION}

Over the last 2 decades a lot of efforts has been put into understanding the interactions between lipids and $\mathrm{Si}-\mathrm{Hy}$ lenses. Most studies were focused on the evaluation of lipid deposits on the surface and in the lens matrix. Even though contact lenses have been extensively tested in this direction since the 1970s, the introduction of new Si-Hy materials, with changed chemical characteristics, made new tests necessary.

Reviewing the studies, three types of studies can be found:

1. In vivo, least common, with the use of a biomicroscope and a grading scale. Only one such study was found in this literature review.

2. Ex vivo, in which worn lenses obtained from users were tested outside of the eye environment. This type of research gives some limitations to the comparison of lens properties and their interaction with lipids because of differences in tears lipid chemical compositions by race [75] and individuals [44, 99].

3. In vitro, in which unworn lenses were treated and then tested. With the development of these studies, efforts were made to reflect as much as possible the conditions in situ, for example by creating a multi-component AT $[59,67,69,76]$, or mechanisms that imitate blinking and tear flow $[68,87,93]$.

Different methods used in studies are presented in table 1. Some of the studies quantified lipids deposited onto lenses; other aimed to visualize deposits both on the surface and in the lens matrix.

\begin{tabular}{|c|c|c|}
\hline \multicolumn{3}{|c|}{$\begin{array}{l}\text { Methods used to evaluate lipids on lenses found in reviewed } \\
\text { studies. }\end{array}$} \\
\hline & Methods & Refs. \\
\hline \multirow{11}{*}{ 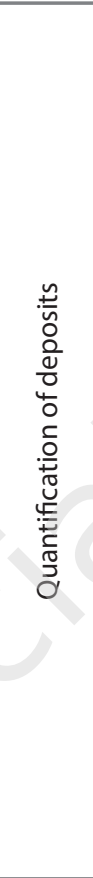 } & $\begin{array}{l}\text { high-performance liquid } \\
\text { chromatography }\end{array}$ & {$[16,43,79]$} \\
\hline & thin layer chromatography & {$[42,51,61,62,91]$} \\
\hline & $\begin{array}{l}\text { gas chromatography/mass } \\
\text { spectrometry GC/MS }\end{array}$ & [47] \\
\hline & fluorescence spectroscopy & {$[48,61]$} \\
\hline & $\begin{array}{c}\text { cholesterol esterase enzymatic } \\
\text { reaction }\end{array}$ & [53] \\
\hline & fluorometric enzymatic assay & {$[55,73,77,97]$} \\
\hline & $\begin{array}{l}\text { electrospray ionization tandem } \\
\text { mass spectrometry (ESI-MS/MS) }\end{array}$ & {$[56,66,87]$} \\
\hline & $\begin{array}{l}\text { beta counter, scintillation } \\
\text { counting }\end{array}$ & $\begin{array}{c}{[58,63,67,68,69,} \\
71,74,76,82,85, \\
86,94,95] \\
\end{array}$ \\
\hline & $\begin{array}{l}\text { normal phase high-performance } \\
\text { liquid chromatography }\end{array}$ & [60] \\
\hline & $\begin{array}{l}\text { liquid extraction surface analysis } \\
\& \text { tandem mass spectrometry } \\
\text { (LESA-MS/MS) }\end{array}$ & [72] \\
\hline & $\begin{array}{l}\text { liquid chromatography-mass } \\
\text { spectrometry technique }\end{array}$ & [98] \\
\hline \multirow{4}{*}{ 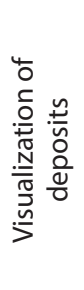 } & atomic force microscopy & {$[46,80]$} \\
\hline & fluorescence microscopy & {$[64,84]$} \\
\hline & $\begin{array}{c}\text { fluorescence confocal scanning } \\
\text { laser microscopy }\end{array}$ & {$[87,93,96]$} \\
\hline & optical microscopy & [87] \\
\hline
\end{tabular}

Most studies (both ex vivo and in vitro) which aimed to measure the amount of lipid deposits on Si-Hy lenses required lipid extraction from the lenses. Best solution for that purpose is $2: 1$ chloroform : methanol [42].

Numerous studies have confirmed that the modified material chemistry of $\mathrm{Si}-\mathrm{Hy}$ vs. hydrogel lenses has influenced the type and amount of deposits, manifesting itself primarily in a higher amount of lipid deposits. Moreover, the amount of deposits depends on Si-Hy lens materials and surface treatment [12, 16, 43, 51, 55, 77, 95]. Differences in deposits on Si-Hy lenses were also observed between polar and non-polar lipids [56]. In general, there are significantly different results in the literature as to the amount of lipid deposits on lenses. These differences may be mainly related to significant differences in the materials and the measurement methods used. 
With regard to the mechanisms of deposit formation, studies show that they can be observed even after $2 \mathrm{~h}$ [86] of incubation in a lipid solution or the first days of wearing [54]. A visualization-oriented study showed that Nile Red $\mathrm{O}$ is an efficient stain for the visualization of lipid deposits [64]. Additionally, the research using fluorescence confocal scanning laser microscopy showed that after some time lipid deposits in the lens matrix reach a homogeneous distribution [96].

A majority of the research analyzed lipids from the tear film. However, there have also been studies with lipids originating from hands that have shown transfer of lipids to lenses while manipulating them and highlighting the importance of proper hand washing $[47,61]$.

Interestingly, one study showed that lipid deposits in $\mathrm{Si}-\mathrm{Hy}$ lenses are degrading during sleep. The consequence of lipid degradation is the production of oxidative products, which may be linked to contact lens discomfort [78].

Despite the commonly acknowledged influence of lipid deposits on discomfort in contact lenses, it is difficult to find direct evidence for this in the literature. Moreover, some studies indicate the opposite, e.g., that lipid deposits on $\mathrm{Si}-\mathrm{Hy}$ lenses can improve lens wettability and comfort [45]. Another study showed a weak correlation between clinical response and the amount of lipid deposits [57]. One recent study showed that asymptomatic contact lens wearers have more deposits on their lenses than symptomatic wearers [98].

The positive effect of one of the lipids, namely phosphatidylcholine, on the stability of the tear film is well-known. The researchers decided to take this fact into consideration and investigate if this lipid can be loaded onto a Si-Hy lens and the process of releasing it into the tear film in time. The results showed the ease of loading and controllable release of DMPC from Si-Hy, presenting the possibility of using such lenses to counter eye discomfort caused by inherently low levels of phospholipids in tears $[63,70]$.

Another direction of research was to check whether lipid deposits can affect the adhesion of bacteria to lenses. Studies have shown that neither cholesterol nor phospholipid deposits affect the adhesion of bacteria $[62,66]$.

Many studies have also covered the interaction of lipid deposits, $\mathrm{Si}$-Hy lenses and care solutions. It was proved that the amount of lipid deposits does not affect the rate of neutralizing peroxide solutions. The next studies checked whether the solutions clean the lenses from lipid deposits, because the early results did not demonstrate such properties of care solutions $[68,81]$. Only the latest study showed that cholesterol sorption was significantly lower when subjects used care solutions with EOBO [97].

\section{CONCLUSION}

The review of the perused literature shows the complexity of the interactions between lipids and $\mathrm{Si}-\mathrm{Hy}$ contact lenses. Over the last 2 decades, mainly lipid deposits on such lenses have been studied, and although there have been significant deviations in the results, some regularities have been observed, such as:

- Si-Hy lenses have a higher tendency to attract and accumulate lipids than traditional hydrogel lenses.

- Different Si-Hy lens materials exhibited different properties for lipid deposits, which could result from the properties of the polymers or the surface treatment.

Moreover, no standard method to assess the amount of lipid deposits in $\mathrm{Si}-\mathrm{Hy}$ lenses was proposed up to now what could lead to discrepancies between the results. As a result, the effects of lipid deposits on Si-Hy lenses on patients' clinical response, in particular, their impact on discomfort are not fully understand. Nevertheless, understanding the role of polar lipids in tear film has led to the development of a technology to release phosphatidylcholine from contact lenses in order to improve the stability of the tear film during lens wear.

The ratio of lipid deposits on Si-Hy lens surfaces to the lipids deposited in the lens matrix could not be accurately determined and, thus, contrasted with the clinical response of the patients. They can only be visualized.

On the basis of the state-of-the-art knowledge on the topic one can propose further directions of research:

- Determination of physicochemical phenomena responsible for the bonding of lipids to the surface and matrix of lenses.

- Evaluation of the rate of lipid deposition on lenses in the first seconds and minutes after contact with lipid-containing solution. The movement of lipids inside the lenses is unknown. This could be examined using FRAP (fluorescence recovery after photobleaching) method, previously used by Suliński and Gapiński to study diffusion in $\mathrm{Si}-\mathrm{Hy}$ lens matrix (example of FRAP recording in figure 7) [100].

- Determination of the processes that are involved in care solutions acting on lipids on the surface and in the Si-Hy lens matrix.

- Verification if the interactions of Si-Hy lenses with lipids are influenced by the composition of the solution in the lens blister, which may be particularly important for daily disposable lenses.

- Investigation of the interactions of lipids from wetting drops with Si-Hy lenses. Despite the common knowledge that wetting drops should not be used with lipids when using lenses, there is no literature on the subject apart from indications from manufacturers. 


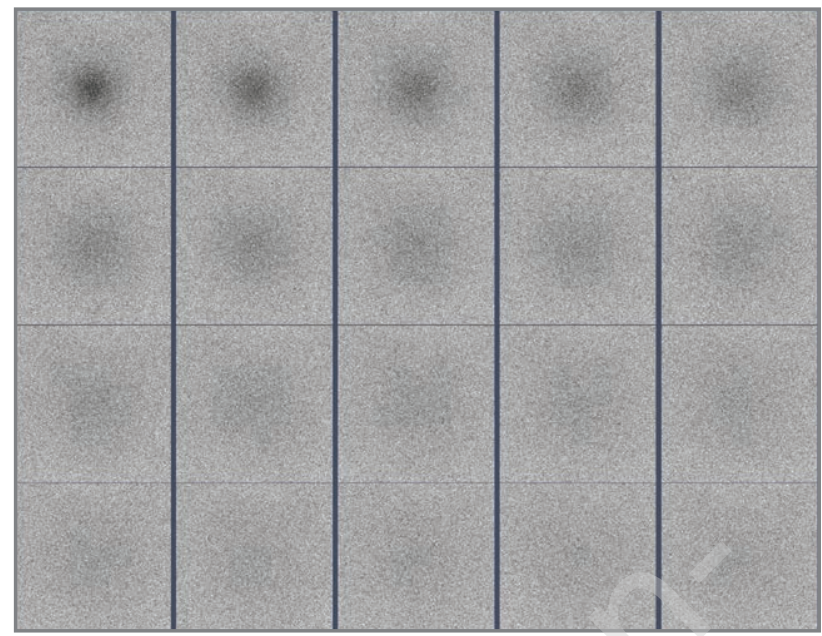

Figures: from the author's own materials.

\section{CORRESPONDENCE}

Tomasz Suliński, MSC

Alcon Polska, Poland

02-674 Warsaw, Marynarska 15

\section{ORCID}

e-mail: tomasz.sulinski@alcon.com

\footnotetext{
Tomasz Suliński - ID - http://orcid.org/0000-0001-9629-3562 Jacek Pniewski - ID - http://orcid.org/0000-0002-6169-120X
}

\section{References}

1. Efron N. History. In: Contact Lens Practice. 2018: 7. http://doi.org/10.1016/b978-0-7020-6660-3.00001-0.

2. Morgan PB, Woods CA, Tranoudis IG et al. Contact Lens Spectrum - International Contact Lens Prescribing in 2014. Contact Lens Spectr. 2015; 30(January 2015): 28-33. https://www.clspectrum.com/issues/2020/january-2020/international-contact-lens-prescribing-in-2019 (access: 9.09.2020).

3. Yokoi N, Bron AJ, Georgiev GA. The precorneal tear film as a fluid shell: The effect of blinking and saccades on tear film distribution and dynamics. Ocul Surf. 2014; 12(4): 252-66. http://doi.org/10.1016/j.jtos.2014.01.006.

4. McCulley JP, Shine W. A compositional based model for the tear film lipid layer. Trans Am Ophthalmol Soc. 1997; 95: 79-93.

5. King-Smith PE, Bailey MD, Braun RJ. Four characteristics and a model of an effective tear film lipid layer (tfll). Ocul Surf. 2013; 11(4): 236-45. http://doi.org/10.1016/j.jtos.2013.05.003.

6. Bron AJ, Tiffany JM, Gouveia SM et al. Functional aspects of the tear film lipid layer. Exp Eye Res. 2004; 78(3): 347-60. http://doi. org/10.1016/j.exer.2003.09.019.

7. Peng CC, Cerretani C, Li Y et al. Flow evaporimeter to assess evaporative resistance of human tear-film lipid layer. Ind Eng Chem Res. 2014; 53(47): 18130-9. http://doi.org/10.1021/ie5030497.

8. Craig JP, Tomlinson A. Importance of the lipid layer in human tear film stability and evaporation. Optom Vis Sci. 1997; 74(1): 8-13. http://doi.org/10.1097/00006324-199701000-00014.

9. Brown SHJ, Kunnen CME, Duchoslav E et al. A comparison of patient matched meibum and tear lipidomes. Investig Ophthalmol Vis Sci. 2013; 54(12): 7417-23. http://doi.org/10.1167/iovs.13-12916.

10. Millar TJ, Schuett BS. The real reason for having a meibomian lipid layer covering the outer surface of the tear film - A review. Exp Eye Res. 2015; 137: 125-38. http://doi.org/10.1016/j.exer.2015.05.002.

11. Lam SM, Tong L, Reux B et al. Lipidomic analysis of human tear fl uid reveals structure-specific lipid alterations in dry eye syndrome. J Lipid Res. 2014; 55(2): 299-306. http://doi.org/10.1194/jlr.P041780.

12. Cheung SW, Cho P, Chan B et al. A comparative study of biweekly disposable contact lenses: Silicone hydrogel versus hydrogel. Clin Exp Optom. Published online 2007. http://doi.org/10.1111/j.1444-0938.2006.00107.x. 
13. Nichols JJ. Deposition on silicone hydrogel lenses. Eye Contact Lens. 2013; 39(1): 20-3. http://doi.org/10.1097//CL.0b013e318275305b.

14. Tighe BJ. A Decade of Silicone Hydrogel Development. Eye Contact Lens Sci Clin Pract. 2013; 39(1): 1. http://doi.org/10.1097/ ICL.0b013e318275452b.

15. Lorentz H, Jones L. Lipid Deposition on Hydrogel Contact Lenses: How History Can Help Us Today. Optom Vis Sci. 2007; 84(4): $286-95$. http://doi.org/10.1097/OPX.0b013e3180485d4b.

16. Jones L, Senchyna M, Glasier MA et al. Lysozyme and lipid deposition on silicone hydrogel contact lens materials. Eye Contact Lens. 2003; 29(1 suppl). http://doi.org/10.1097/00140068-200301001-00021.

17. Nichols JJ, Willcox MDP, Bron AJ et al. The TFOS International Workshop on Contact Lens Discomfort: Executive summary. Investig Ophthalmol Vis Sci. 2013; 54(11): TFOS7-TFOS13. http://doi.org/10.1167/iovs.13-13212.

18. Doughman DJ, Mobilia E, Drago D et al. The nature of "spots" on soft lenses. Ann Ophthalmol. 1975; 7(3): 345-8, 351-3.

19. Wedler FC. Analysis of biomaterials deposited on soft contact lenses. J Biomed Mater Res. 1977; 11(4):525-35. http://doi.org/10.1002/ jbm.820110408.

20. Binder PS. Complications Associated With Extended Wear of Soft Contact Lenses. Ophthalmology. 1979; 86(6): 1093-101. http://doi. org/10.1016/S0161-6420(79)35420-3.

21. Rubey F. [Possibilities of examination on soft contact lenses (author's transl)]. Klin Monbl Augenheilkd. 1978; 172(2): $222-5$.

22. Hart DE, Tidsale RR, Sack RA. Origin and Composition of Lipid Deposits on Soft Contact Lenses. Ophthalmology. 1986; 93(4): 495-503. http://doi.org/10.1016/S0161-6420(86)33709-6.

23. Tripathi RC, Tripathi BJ, Ruben M. The Pathology of Soft Contact Lens Spoilage. Ophthalmology. 1980; 87(5): 365-80. http://doi. org/10.1016/S0161-6420(80)35222-6.

24. Hosaka S, Ozawa H, Tanzawa $\mathrm{H}$ et al. Analysis of deposits on high water content contact lenses. J Biomed Mater Res. 1983; 17(2): 261-74. http://doi.org/10.1002/jbm.820170205.

25. Lane BC. Spoliage of Hydrogel Contact Lenses by Lipid Deposits: Tear-film Potassium Depression, Fat, Protein, and Alcohol Consumption. Ophthalmology. 1987; 94(10): 1315-21. http://doi.org/10.1016/S0161-6420(87)80018-0.

26. Magran BL, Hurtado I. Soft contact lens damage: a one-year study in Caracas, Venezuela. CLAO J Off Publ Contact Lens Assoc Ophthalmol Inc. 1989; 15(4): 274-8.

27. Rapp J, Broich J. Lipid Deposits on Worn Soft Contact Lenses. CLAO J. 1984; 10(3): 235-40.

28. Caroline PJ, Robin JB, Gindi JJ et al. Microscopic and elemental analysis of deposits on extended wear soft contact lenses. CLAO J Off Publ Contact Lens Assoc Ophthalmol Inc. 1985; 11(4): 311-6.

29. Bowers RWJ, Tighe BJ. Studies of the ocular compatibility of hydrogels. White spot deposits-chemical composition and geological arrangement of components. Biomaterials. 1987; 8(3). http://doi.org/10.1016/0142-9612(87)90059-7.

30. Begley CG, Waggoner PJ. An analysis of nodular deposits on soft contact lenses. J Am Optom Assoc. 1991; 62(3): 208-14.

31. Franklin V, Horne A, Jones $L$ et al. Early deposition trends on group I (Polymacon and Tetrafilcon A) and group III (Bufilcon A) materials. CLAO J Off Publ Contact Lens Assoc Ophthalmol Inc. 1991; 17(4): 244-8.

32. Mirejovsky D, Patel AS, Rodriguez DD et al. Lipid adsorption onto hydrogel contact lens materials. advantages of nile red over oil red o in visualization of lipids. Optom Vis Sci. 1991; 68(11): 858-64. http://doi.org/10.1097/00006324-199111000-00005.

33. Tripathi RC, Tripathi BJ, Silverman RA et al. Contact lens deposits and spoilage: Identification and management. Int Ophthalmol Clin. 1991; 31(2): 91-120. http://doi.org/10.1097/00004397-199103120-00012.

34. Bontempo AR, Rapp J. Lipid deposits on hydrophilic and rigid gas permeable contact lenses. Eye Contact Lens. 1994; 20 (4): $242-5$. http://doi.org/10.1097/00140068-199410000-00009.

35. Ho CH, Hlady V. Fluorescence assay for measuring lipid deposits on contact lens surfaces. Biomaterials. 1995; 16(6): 479-82. http:// doi.org/10.1016/0142-9612(95)98821-U.

36. Jones L, Franklin V, Evans K et al. Spoilation and Clinical Performance of Monthly vs. Three Monthly Group II Disposable Contact Lenses. Optom Vis Sci. 1996; 73(1): 16-21. http://doi.org/10.1097/00006324-199601000-00003.

37. Jones L, Evans K, Sariri R et al. Lipid and protein deposition of N-vinyl pyrrolidone-containing group II and group IV frequent replacement contact lenses. CLAO J Off Publ Contact Lens Assoc Ophthalmol Inc. 1997; 23(2): 122-6.

38. Bontempo AR, Rapp J. Protein-lipid interaction on the surface of a hydrophilic contact lens in vitro. Curr Eye Res. 1997; 16(8): 776-81. http://doi.org/10.1076/ceyr.16.8.776.8985.

39. Prager MD, Quintana RP. Radiochemical studies on contact lens soilation. II. Lens uptake of cholesteryl oleate and dioleoyl phosphatidylcholine. J Biomed Mater Res. 1997; 37(2): 207-11. http://doi.org/10.1002/(SICI)1097-4636(199711)37:2<207::AID-JBM9>3.0.CO;2-V.

40. Maïssa C, Franklin V, Guillon M et al. Influence of contact lens material surface characteristics and replacement frequency on protein and lipid deposition. Optom Vis Sci. 1998; 75(9): 697-705. http://doi.org/10.1097/00006324-199809000-00026.

41. Hart E, Guillon M. Influence of contact lens material surface characteristics and replacement frequency on protein and lipid deposition (multiple letters). Optom Vis Sci. 1999; 76(9): 616-7. http://doi.org/10.1097/00006324-199909000-00016. 
42. Lornetz H, Senchyna M, Jones L. Optimized Procedure for the Extraction of Lipid Deposits from Silicone-Hydrogel Contact Lenses. IOVS | ARVO Journals. 2004; 45(13): 1537. https://iovs.arvojournals.org/article.aspx?articleid=2407103 (access: 26.10.2020).

43. Maziarz EP, Stachowski MJ, Liu XM et al. Lipid Deposition on Silicone Hydrogel Lenses, Part I: Quantification of Oleic Acid, Oleic Acid Methyl Ester, and Cholesterol. Eye Contact Lens Sci Clin Pract. 2006; 32(6): 300-7. http://doi.org/10.1097/01.icl.0000224365.51872.6c.

44. Jones L, Subbaraman L, Rogers R et al. Surface treatment, wetting and modulus of silicone hydrogels. Optician. 2006; 232: 28-34.

45. Lorentz H, Rogers R, Jones L. The impact of lipid on contact angle wettability. Optom Vis Sci. 2007; 84(10): 946-53. http://doi. org/10.1097/OPX.0b013e318157a6c1.

46. Lira M, Santos L, Azeredo J et al; Real Oliveira MECD. Comparative study of silicone-hydrogel contact lenses surfaces before and after wear using atomic force microscopy. J Biomed Mater Res - Part B Appl Biomater. 2008; 85(2): 361-7. http://doi.org/10.1002/ jbm.b.30954.

47. Iwata M, Ohno S, Kawai T et al. In vitro evaluation of lipids adsorbed on silicone hydrogel contact lenses using a new gas chromatography/mass spectrometry analytical method. Eye Contact Lens. 2008; 34(5): 272-80. http://doi.org/10.1097/icl.0b013e318182f357.

48. Carney FP, Nash WL, Sentell KB. The adsorption of major tear film lipids in vitro to various silicone hydrogels over time. Investig Ophthalmol Vis Sci. 2008; 49(1): 120-4. http://doi.org/10.1167/iovs.07-0376.

49. Jones L, Senchyna M, Glasier M et al. Lysozyme and lipid deposition on silicone hydrogel contact lens materials. journals.Iww.com. https://journals.Iww.com/claojournal/Fulltext/2003/01001/Lysozyme_and_Lipid_Deposition_on_Silicone_Hydrogel.21.aspx (access: 11.09.2020).

50. Ngo W, Heynen M, Joyce E et al. Impact of protein and lipid on neutralization times of hydrogen peroxide care regimens. Eye Contact Lens. 2009; 35(6): 282-6. http://doi.org/10.1097//CL.0b013e3181b93bd1.

51. Zhao Z, Carnt NA, Aliwarga $Y$ et al. Care regimen and lens material influence on silicone hydrogel contact lens deposition. Optom Vis Sci. 2009; 86(3): 251-9. http://doi.org/10.1097/OPX.0b013e318196a74b.

52. Svitova TF, Lin MC. Tear lipids interfacial rheology: Effect of lysozyme and lens care solutions. Optom Vis Sci. 2010; 87(1): 10-20. http:// doi.org/10.1097/OPX.0b013e3181c07908.

53. Pucker AD, Thangavelu M, Nichols JJ. Enzymatic quantification of cholesterol and cholesterol esters from silicone hydrogel contact lenses. Investig Ophthalmol Vis Sci. 2010; 51(6): 2949-54. http://doi.org/10.1167/iovs.08-3368.

54. Pucker AD, Thangavelu M, Nichols JJ. In vitro lipid deposition on hydrogel and silicone hydrogel contact lenses. Investig Ophthalmol Vis Sci. 2010; 51(12): 6334-40. http://doi.org/10.1167/iovs.10-5836.

55. Walther N, Gabriel M, Mowrey-McKee M. A comparison of various silicone hydrogel lenses; lipid and protein deposition as a result of daily wear. https://www.aaopt.org/detail/knowledge-base-article/comparison-various-silicone-hydrogel-lenses-lipid-and-protein-deposition-result-daily-wear (access: 9.09.2020).

56. Saville JT, Zhao Z, Willcox MDP et al. Detection and quantification of tear phospholipids and cholesterol in contact lens deposits: The effect of contact lens material and lens care solution. Investig Ophthalmol Vis Sci. 2010; 51(6): 2843-51. http://doi.org/10.1167/ iovs.09-4609.

57. Zhao Z, Naduvilath T, Flanagan JL et al. Contact lens deposits, adverse responses, and clinical ocular surface parameters. Optom Vis Sci. 2010; 87(9): 669-74. http://doi.org/10.1097/OPX.0b013e3181ea1848.

58. Walther $\mathrm{H}$, Lorentz $\mathrm{H}$, Kay $\mathrm{L}$ et al. 20 The effect of in vitro lipid concentration on lipid deposition on silicone hydrogeland conventional hydrogel contact lens materials. Contact Lens Anterior Eye. 2011; 34: S21. http://doi.org/10.1016/s1367-0484(11)60099-4.

59. Lorentz H, Heynen M, Kay LMM et al. Contact lens physical properties and lipid deposition in a novel characterized artificial tear solution. Mol Vis. 2011; 17: 3392-405.

60. Heynen M, Lorentz H, Srinivasan S et al. Quantification of non-polar lipid deposits on senofilcon A Contact Lenses. Optom Vis Sci. 2011; 88(10): 1172-9. http://doi.org/10.1097/OPX.0b013e31822a5295.

61. Campbell D, Mann A, Hunt $\mathrm{O}$ et al. The significance of hand wash compliance on the transfer of dermal lipids in contact lens wear. Contact Lens Anterior Eye. 2012; 35(2): 71-6. http://doi.org/10.1016/j.clae.2011.11.004.

62. Omali NB, Zhu H, Zhao Z et al. Effect of cholesterol deposition on bacterial adhesion to contact lenses. Optom Vis Sci. 2011; 88(8): 950-8. http://doi.org/10.1097/OPX.0b013e31821cc683.

63. Pitt WG, Jack DR, Zhao Y et al. Loading and release of a phospholipid from contact lenses. Optom Vis Sci. 2011; 88(4): 502-6. http:// doi.org/10.1097/OPX.0b013e31820e9ff8.

64. Pucker AD, Nichols JJ. A method of imaging lipids on silicone hydrogel contact lenses. Optom Vis Sci. 2012; 89(5). http://doi. org/10.1097/OPX.0b013e318253dea9.

65. Vishnubhatla S, Borchman D, Foulks GN. Contact lenses and the rate of evaporation measured in vitro; the influence of wear, squalene and wax. Contact Lens Anterior Eye. 2012; 35(6): 277-81. http://doi.org/10.1016/j.clae.2012.07.008.

66. Babaei Omali N, Proschogo N, Zhu H et al. Effect of phospholipid deposits on adhesion of bacteria to contact lenses. Optom Vis Sci. 2012; 89(1): 52-61. http://doi.org/10.1097/OPX.0b013e318238284c. 
67. $\mathrm{Ng} \mathrm{A}$, Heynen M, Luensmann D et al. Impact of tear film components on lysozyme deposition to contact lenses. Optom Vis Sci. 2012; 89(4): 392-400. http://doi.org/10.1097/OPX.0b013e31824c0c4a.

68. Lorentz H, Heynen M, Khan W et al. The impact of intermittent air exposure on lipid deposition. Optom Vis Sci. 2012; 89(11): $1574-81$. http://doi.org/10.1097/OPX.0b013e31826c6508.

69. Lorentz H, Heynen M, Trieu D et al. The impact of tear film components on in vitro lipid uptake. Optom Vis Sci. 2012; 89(6): 856-67. http://doi.org/10.1097/OPX.0b013e318255ddc8.

70. Pitt WG, Jack DR, Zhao Y et al. Transport of phospholipid in silicone hydrogel contact lenses. J Biomater Sci Polym Ed. 2012; 23(1-4): 527-41. http://doi.org/10.1163/092050611X554174.

71. Lorentz $\mathrm{H}$, Heynen M, Tran $\mathrm{H}$ et al. Using an in vitro model of lipid deposition to assess the efficiency of hydrogen peroxide solutions to remove lipid from various contact lens materials. Curr Eye Res. 2012; 37(9): 777-86. http://doi.org/10.3109/02713683.2012.682636.

72. Brown SHJ, Huxtable LH, Willcox MDP et al. Automated surface sampling of lipids from worn contact lenses coupled with tandem mass spectrometry. Analyst. 2013; 138(5): 1316-20. http://doi.org/10.1039/c2an36189b.

73. $\mathrm{Ng} \mathrm{A}$, Heynen $\mathrm{M}$, Luensmann D et al. Impact of tear film components on the conformational state of lysozyme deposited on contact lenses. J Biomed Mater Res - Part B Appl Biomater. 2013; 101(7): 1172-81. http://doi.org/10.1002/jbm.b.32927.

74. Pitt WG, Perez KX, Tam NK et al. Quantitation of cholesterol and phospholipid sorption on silicone hydrogel contact lenses. J Biomed Mater Res - Part B Appl Biomater. 2013; 101(8): 1516-23. http://doi.org/10.1002/jbm.b.32973.

75. Svitova TF, Lin MC. Racial variations in interfacial behavior of lipids extracted from worn soft contact lenses. Optom Vis Sci. 2013; 90(12): 1361-9. http://doi.org/10.1097/OPX.0000000000000098.

76. Walther $\mathrm{H}$, Lorentz $\mathrm{H}$, Heynen $\mathrm{M}$ et al. Factors that influence in vitro cholesterol deposition on contact lenses. Optom Vis Sci. 2013; 90(10): 1057-65. http://doi.org/10.1097/OPX.0000000000000022.

77. Nash WL, Gabriel MM. Ex vivo analysis of cholesterol deposition for commercially available silicone hydrogel contact lenses using a fluorometric enzymatic assay. Eye Contact Lens. 2014; 40(5): 277-82. http://doi.org/10.1097//CL.0000000000000052.

78. Panaser A, Tighe BJ. Evidence of lipid degradation during overnight contact lens wear: Gas chromatography mass spectrometry as the diagnostic tool. Investig Ophthalmol Vis Sci. 2014; 55(3): 1797-804. http://doi.org/10.1167/iovs.13-12881.

79. Maissa C, Guillon M, Cockshott N et al. Contact lens lipid spoliation of hydrogel and silicone hydrogel lenses. Optom Vis Sci. 2014; 91(9): 1071-83. http://doi.org/10.1097/OPX.0000000000000341.

80. Cheung S, Lorentz H, Drolle E et al. Comparative study of lens solutions' ability to remove tear constituents. Optom Vis Sci. 2014; 91(9): 1045-61. http://doi.org/10.1097/OPX.0000000000000340.

81. Tam NK, Pitt WG, Perez KX et al. The role of multi-purpose solutions in prevention and removal of lipid depositions on contact lenses. Contact Lens Anterior Eye. 2014; 37(6): 405-14. http://doi.org/10.1016/j.clae.2014.07.003.

82. Tam NK, Pitt WG, Perez KX et al. Prevention and removal of lipid deposits by lens care solutions and rubbing. Optom Vis Sci. 2014; 91(12): 1430-9. http://doi.org/10.1097/OPX.0000000000000419.

83. Bhamla MS, Nash WL, Elliott S et al. Influence of lipid coatings on surface wettability characteristics of silicone hydrogels. Langmuir. 2015; 31(13): 3820-8. http://doi.org/10.1021/la503437a.

84. Hagedorn S, Drolle E, Lorentz H et al. Atomic force microscopy and Langmuir-Blodgett monolayer technique to assess contact lens deposits and human meibum extracts. J Optom. 2015; 8(3): 187-99. http://doi.org/10.1016/j.optom.2014.12.003.

85. Pitt WG, Zhao Y, Jack DR et al. Extended elution of phospholipid from silicone hydrogel contact lenses. J Biomater Sci Polym Ed. 2015; 26(4): 224-34. http://doi.org/10.1080/09205063.2014.994947.

86. Walther H, Subbaraman L, Jones LW. In vitro cholesterol deposition on daily disposable contact lens materials. Optom Vis Sci. 2016; 93(1): 36-41. http://doi.org/10.1097/OPX.0000000000000749.

87. Peng C-C, Fajardo NP, Razunguzwa T et al. In Vitro Spoilation of Silicone-Hydrogel Soft Contact Lenses in a Model-Blink Cell. Optom Vis Sci. 2015; 92(7): 768-80. http://doi.org/10.1097/OPX.0000000000000625.

88. Silva D, Fernandes AC, Nunes TG et al. The effect of albumin and cholesterol on the biotribological behavior of hydrogels for contact lenses. Acta Biomater. 2015; 26: 184-94. http://doi.org/10.1016/j.actbio.2015.08.011.

89. Bassyouni RH, Kamel Z, Abdelfattah MM et al. Cinnamon oil: A possible alternative for contact lens disinfection. Contact Lens Anterior Eye. 2016; 39(4): 277-83. http://doi.org/10.1016/j.clae.2016.01.001.

90. Wang MTM, Ganesalingam K, Loh CS et al. Compatibility of phospholipid liposomal spray with silicone hydrogel contact lens wear. Contact Lens Anterior Eye. 2017; 40(1): 53-8. http://doi.org/10.1016/j.clae.2016.11.002.

91. Schuett BS, Millar TJ. An Experimental Model to Study the Impact of Lipid Oxidation on Contact Lens Deposition In Vitro. Curr Eye Res. 2017; 42(9): 1220-7. http://doi.org/10.1080/02713683.2017.1307416.

92. Babaei Omali N, Lada M, Lakkis C et al. Lipid Deposition on Contact Lenses when Using Contemporary Care Solutions. Optom Vis Sci. 2017; 94(9): 919-27. http://doi.org/10.1097/OPX.0000000000001114. 
93. Walther H, Phan CM, Subbaraman LN et al. Differential deposition of fluorescently tagged cholesterol on commercial contact lenses using a novel in vitro eye model. TransI Vis Sci Technol. 2018; 7(2). http://doi.org/10.1167/tvst.7.2.18.

94. Walther H, Subbaraman LN, Jones L. Efficacy of Contact Lens Care Solutions in Removing Cholesterol Deposits from Silicone Hydrogel Contact Lenses. Eye Contact Lens. 2019; 45(2): 105-11. http://doi.org/10.1097/ICL.0000000000000547.

95. Luensmann D, Omali NB, Suko A et al. Kinetic Deposition of Polar and Non-polar Lipids on Silicone Hydrogel Contact Lenses. Curr Eye Res. 2020; 1-7 http://doi.org/10.1080/02713683.2020.1755696.

96. Qiao H, Luensmann D, Heynen $\mathrm{M}$ et al. In vitro evaluation of the location of cholesteryl ester deposits on monthly replacement silicone hydrogel contact lens materials. Clin Ophthalmol. 2020; 14: 2821-8. http://doi.org/10.2147/OPTH.S270575.

97. Shows A, Redfern RL, Sickenberger W et al. Lipid Analysis on Block Copolymer-containing Packaging Solution and Lens Care Regimens: A Randomized Clinical Trial. Optom Vis Sci. 2020; 97(8): 565-72. http://doi.org/10.1097/OPX.0000000000001553.

98. Omali NB, Subbaraman LN, Heynen M et al. Lipid deposition on contact lenses in symptomatic and asymptomatic contact lens wearers. Contact Lens Anterior Eye. Published online 2020. http://doi.org/10.1016/j.clae.2020.05.006.

99. Brown SHJ, Kunnen CME, Papas EB et al. Intersubject and Interday Variability in Human Tear and Meibum Lipidomes: A Pilot Study. Ocul Surf. 2016; 14(1): 43-8. http://doi.org/10.1016/j.jtos.2015.08.005.

100. Suliński T, Gapiński J. Badanie barier dyfuzyjnych w polimerowo-hydrożelowych matrycach na przykładzie dyfuzji wybranego leku znieczulajacego w sylikonowo-hydrożelowych soczewkach kontaktowych. Optyka. 2013; 6(25): 36-41.

Authors' contributions:

All authors have the same contribution.

Conflict of interest:

Tomasz Suliński is an employee of the Alcon.

Financial support:

Tomasz Suliński receives remuneration for his work in the Alcon.

Ethics:

The content presented in the article complies with the principles of the Helsinki

Declaration, EU directives and harmonized requirements for biomedical journals. 